\title{
Direction Selectivity of Neurons in the Macaque Lateral Intraparietal Area
}

\author{
Alessandra Fanini and John A. Assad \\ Department of Neurobiology, Harvard Medical School, Boston, Massachusetts
}

Submitted 9 April 2007; accepted in final form 1 November 2008

Fanini A, Assad JA. Direction selectivity of neurons in the macaque lateral intraparietal area. J Neurophysiol 101: 289-305, 2009. First published November 5, 2008; doi:10.1152/jn.00400.2007. The lateral intraparietal area (LIP) of the macaque is believed to play a role in the allocation of attention and the plan to make saccadic eye movements. Many studies have shown that LIP neurons generally encode the static spatial location demarked by the receptive field (RF). LIP neurons might also provide information about the features of visual stimuli within the RF. For example, LIP receives input from cortical areas in the dorsal visual pathway that contain many direction-selective neurons. Here we examine direction selectivity of LIP neurons. Animals were only required to fixate while motion stimuli appeared in the RF. To avoid spatial confounds, the motion stimuli were patches of randomly arrayed dots that moved with $100 \%$ coherence in eight different directions. We found that the majority (61\%) of LIP neurons were direction selective. The direction tuning was fairly broad, with a median direction-tuning bandwidth of $136^{\circ}$. The average strength of direction selectivity was weaker in LIP than that of other areas of the dorsal visual stream but that difference may be because of the fact that LIP neurons showed a tonic offset in firing whenever a visual stimulus was in the RF, independent of direction. Direction-selective neurons do not seem to constitute a functionally distinct subdivision within LIP, because those neurons had robust, sustained delay-period activity during a memory delayed saccade task. The direction selectivity could also not be explained by asymmetries in the spatial RF, in the hypothetical case that the animals attended to slightly different locations depending on the direction of motion in the RF. Our results show that direction selectivity is a distinct attribute of LIP neurons in addition to spatial encoding.

\section{IN T R O D U C T I O N}

The primate posterior parietal cortex plays a central role in attending to aspects of the visual scene and in using sensory information to guide movement (Andersen 1987; Mesulam 1999). A particularly well-studied area in the posterior parietal lobe of the macaque monkey is the lateral intraparietal area (LIP). Most LIP neurons respond to visual stimuli within a circumscribed receptive field (Ben Hamed et al. 2001; Blatt et al. 1990) but are also modulated by behavioral relevance of visual stimuli and the demands of the task at hand. For example, if a spot of light is flashed on and off within the receptive field (RF), and the animal must make a saccadic eye movement to the remembered location of the flash, many LIP neurons continue to discharge after the stimulus is extinguished, and/or modulate their firing around the time of the saccade (Andersen et al. 1985; Barash et al. 1991; Colby et al. 1996; Janssen and Shadlen 2005). The firing of LIP neurons can be strongly modulated by the general salience of stimuli within the RF (Gottlieb et al. 1998), how the animal responds

Address for reprint requests and other correspondence: J. A. Assad, Dept. of Neurobiology, Harvard Medical School, 220 Longwood Ave., Boston, MA 02115 (E-mail: jassad@hms.harvard.edu). to target stimuli in the RF (Maimon and Assad 2006; Oristaglio et al. 2006; Snyder et al. 1997), or how the animal is rewarded for movements made toward or away from the RF (Platt and Glimcher 1999; Sugrue et al. 2004). Although many questions remain as to the nature of LIP signals, many previous studies have generally shown that LIP neurons encode something about the static spatial location demarked by the RF. Two broad and related possibilities are that LIP neurons may encode a plan to saccade to the RF location (Snyder et al. 2000) or signal the allocation of attention to that location (Bisley and Goldberg 2003; Goldberg et al. 2006).

There is some evidence that LIP neurons are selective for other attributes of visual stimuli besides the spatial location of stimuli. For example, many LIP neurons are selective for the form of visual stimuli within the RF (Sereno and Amador 2006; Sereno and Maunsell 1998), a property that may arise from inputs to LIP from the ventral visual pathway (Blatt et al. 1990; Lewis and Van Essen 2000a). The dorsal visual pathway, including the middle temporal area (MT) and the medial superior temporal area (MST), is a more prominent source of input to LIP (Blatt et al. 1990; Lewis and Van Essen 2000a; Ungerleider and Desimone 1986). MT and MST contain a preponderance of neurons that are selective for the direction of moving stimuli, which raises the possibility that LIP neurons might inherit direction selectivity via inputs from MT and MST. Direction selectivity in LIP could provide a useful complement to the well-described spatial selectivity in LIP. For example, Duhamel et al. (1992) suggested that LIP neurons update their spatial RF in advance of saccadic eye movements; if LIP neurons predict where stimuli are going to be, it makes sense that they would also receive information about which way those stimuli are headed. In addition, direction selectivity in LIP might play a role in feature-base attention (Treue and Martinez Trujillo 1999) or working memory (Zaksas and Pasternak 2006) in the dorsal visual pathway. Nonetheless, direction selectivity is not commonly attributed to LIP, and there have been no systematic studies of direction selectivity in LIP.

We have observed direction selectivity in LIP under certain experimental conditions in our laboratory (Eskandar and Assad 1999, 2002; Williams et al. 2003). However, in those studies, the visual stimuli were not designed to thoroughly examine directional properties in LIP; for example, only two directions of motion were tested. Moreover, the motion was highly relevant to the animals' accomplishing their task at hand, which raises the question of whether direction selectivity is a dynamic quality that is present only when motion is behaviorally relevant (as we found for color selectivity in LIP; Toth and Assad 2002). This caveat also applies to a recent study in which we found direction-

\footnotetext{
The costs of publication of this article were defrayed in part by the payment of page charges. The article must therefore be hereby marked "advertisement" in accordance with 18 U.S.C. Section 1734 solely to indicate this fact.
} 
selective neurons in LIP in animals that had been extensively trained to divide directions into two "artificial" categories (Freedman and Assad 2006).

In this study, we explore in detail the directional properties of LIP neurons. To minimize confounds related to spatial selectivity and to examine the relationship between direction selectivity and spatial selectivity, we used a visual stimulus consisting of randomly arrayed moving dots confined to the RF. In addition, we tested LIP neurons in animals that were passively fixating, with no task requirements related to the motion of the visual stimulus within the RF. We found that a majority of LIP neurons are indeed direction selective and that the direction selectivity differs in some respects from that found in other cortical areas in the dorsal pathway. Our results suggest that direction selectivity is more widespread in parietal cortex than previously appreciated.

\section{METHODS}

Experiments were performed on two male rhesus monkeys ( $\mathrm{Ma}$ caca mulatta, monkey $H, 14 \mathrm{~kg}$, and monkey $R, 12 \mathrm{~kg}$ ). All animal procedures, including surgeries and postoperative care, followed National Institutes of Health and Harvard Medical School guidelines. Monkeys were prepared for experiments by surgical implantation of a custom titanium head-post for head restraint, celux recording cylinder, and monocular scleral search coil (Judge et al. 1980; Robinson 1963) to monitor eye position. The recording chambers were dorsally positioned at stereotaxic coordinates 3 posterior, 10 lateral in the left and right hemisphere on monkeys $H$ and $R$, respectively. The animals sat in a primate chair with their heads restrained during training and recording sessions, which lasted 3-5 h. Water intake was controlled, and each animal was trained to maintain fixation on a small spot for the entire duration of every trial (unless otherwise noted) for juice reward. A computer controlled stimulus presentation and recorded eye-position, neuronal, and behavioral data. Stimuli were presented on a color video monitor $(1,152 \times 870$ resolution, $75-\mathrm{Hz}$ refresh), positioned $57 \mathrm{~cm}$ from the animal. In all tasks, stimuli were achromatic squares presented on a dark gray background $\left(3 \mathrm{~cd} / \mathrm{m}^{2}\right)$.

\section{Main task}

Direction selectivity was tested by the presentation of a circular patch of random dots moving with $100 \%$ coherence. The dots were small squares $\left(0.1^{\circ}\right.$ wide, $\left.28 \mathrm{~cd} / \mathrm{m}^{2}\right)$ presented at a density of 7 dots/deg ${ }^{2}$. On every video refresh frame $(75 \mathrm{~Hz})$, every dot was displaced by a fixed distance dependent on the speed selected. The diameter and location of the patch of dots was adjusted to fill the RF of the neuron under study. In practice, the diameter of the patch varied between $\sim 3$ and $8^{\circ}$.

Each trial began when the monkey directed gaze to the fixation spot. After a 700-ms delay, a sequence of motion stimuli was presented. To separate the neuronal response to motion from the response to the onset of the dot patch, each period of motion was preceded by the onset of the dots and then by a 400-ms interval during which the dots remained stationary. (For $\sim 30$ neurons recorded early in the experiment, a shorter premotion interval, 200 or $250 \mathrm{~ms}$, was used.) The dots moved in one direction for $600 \mathrm{~ms}$, followed by a 400-ms interstimulus interval, during which the screen was blank except for the fixation point. In each trial, this sequence was repeated twice (monkey $R$ ) or three times (monkey $H$ ). The monkey was rewarded for maintaining fixation within a $2^{\circ}$-wide window until the fixation point was extinguished at the end of the sequence; premature fixation breaks were not rewarded. Trials were separated by a 1,000-ms intertrial interval. Eight directions of motion (separated by $45^{\circ}$ and including the cardinal directions) were pseudorandomly interleaved; all eight directions were presented before any single direction was repeated. Typically, 12-16 repetitions of each direction were collected for a given unit.

Most of the cells were tested with more than one speed (maximum 3 ), although examining speed selectivity was not an aim of this study. The speeds tested were $12,25,30$, and $40 \%$ s. The different speeds were tested in separate blocks of trials.

Neither monkey in the study was experimentally naïve. Both animals had been previously trained in a task in which they signaled the start of motion (regardless of direction) of a single dot that comes on, remains stationary, and starts moving. One of the animals had also been trained earlier on a task in which he caused or responded to the reversal of a single moving dot by pressing a lever (Maimon and Assad 2006). The two previous tasks were visually quite different from the main task that we used, and neither task explicitly required the animals to discriminate the direction of motion. In addition, the animals had never paired particular directions of motion with particular directions of eye movements.

\section{Single dot task}

For a subset of cells, following the main task, the direction selectivity was also tested with a single moving white dot $\left(0.75^{\circ}\right.$ wide, 64 $\mathrm{cd} / \mathrm{m}^{2}$ ). On each trial, following fixation and a 700-ms delay, the dot appeared at one of eight locations along the perimeter of an imaginary circle that surrounded the dot patch that had been used for the main task. The dot remained stationary for $400 \mathrm{~ms}$ and started to move in a path that always intersected the point at which the dot patch had been centered. For reasons unrelated to this study, the distance that the dot moved was variable from trial-to-trial, but in all cases, the dot traversed a distance at least as large as the diameter of the dot patch used in the main experiment. The same eight directions of motion that were used in the main task were presented in pseudorandom order, and each direction was repeated 12-16 times. The speed of the dot was set to the speed that elicited the most directional response in the main task.

\section{RF mapping}

Before running the motion tasks, we first determined the spatial extent of the RF for each neuron using a quantitative RF mapping routine based on that of Ben Hamed et al. (2001). To map the RF, the animal fixated while an achromatic square $\left(0.75^{\circ}\right.$ wide, $\left.64 \mathrm{~cd} / \mathrm{m}^{2}\right)$ was briefly flashed at different locations on the screen. The locations that were tested defined an imaginary square matrix covering the region of interest in the visual field. Neuronal responses were monitored online, and the size of the matrix was adjusted depending on the size of the "emerging" RF. The majority of the cells were initially tested with a $6 \times 6$ matrix, with stimulus locations separated horizontally and vertically by $4^{\circ}$ (Fig. $1 A$ ). However, if the preliminary mapping revealed a smaller RF, a higher spatial resolution matrix was additionally tested $(5 \times 5$, with adjacent stimulus locations separated by $2-3^{\circ}$; Fig. $\left.1 B\right)$.

On each trial, following fixation and a 700-ms delay, a sequence of six flashed stimuli was presented. The stimulus locations were chosen pseudorandomly from the 36 (or 25) available locations, and each location was tested before any location was retested. Each stimulus was on for $200 \mathrm{~ms}$, and the interval between successive stimuli was $300 \mathrm{~ms}$. The animal was rewarded for maintaining fixation until the stimulation cycle ended and the fixation point was extinguished. Depending on the neural responsiveness, 6-12 trials were collected for each location of the matrix.

\section{Memory delayed saccade task}

We used a memory delayed saccade task (Hikosaka and Wurtz 1983 ) to characterize $>90 \%$ of the neurons in the study. LIP neurons 


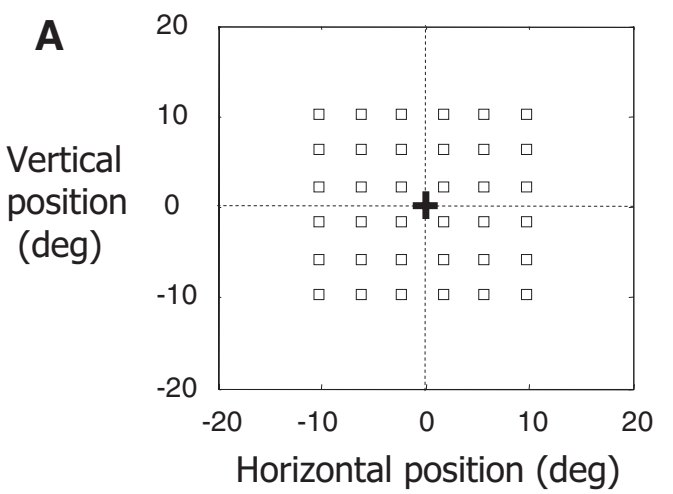

C

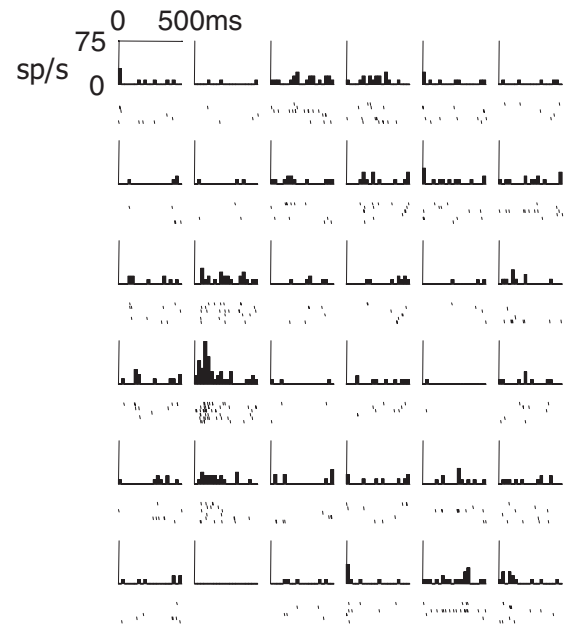

E

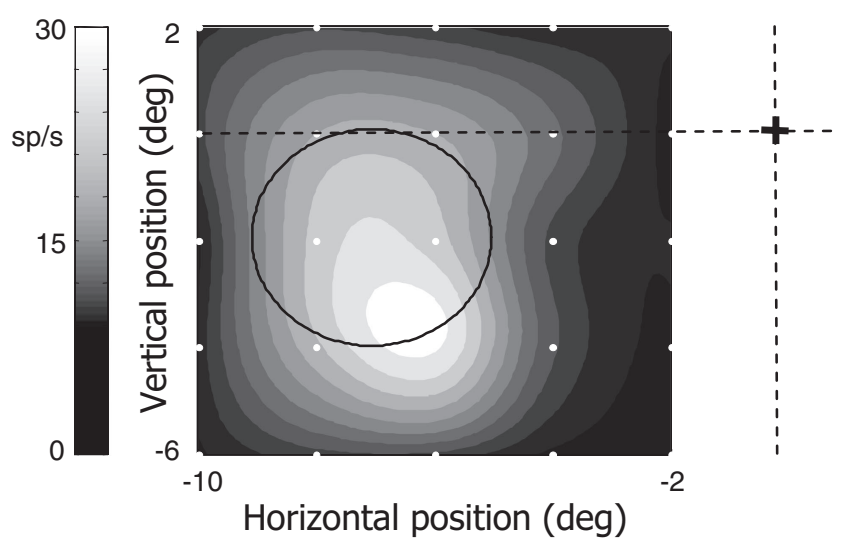

B

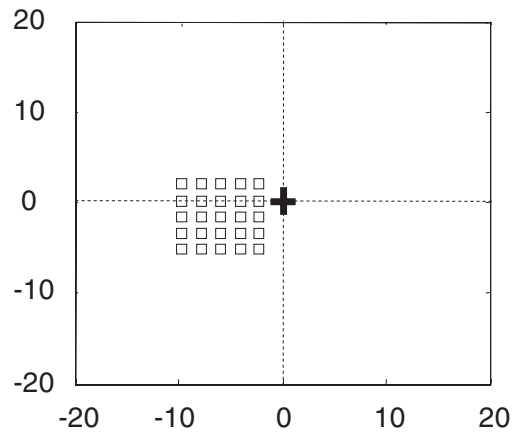

D

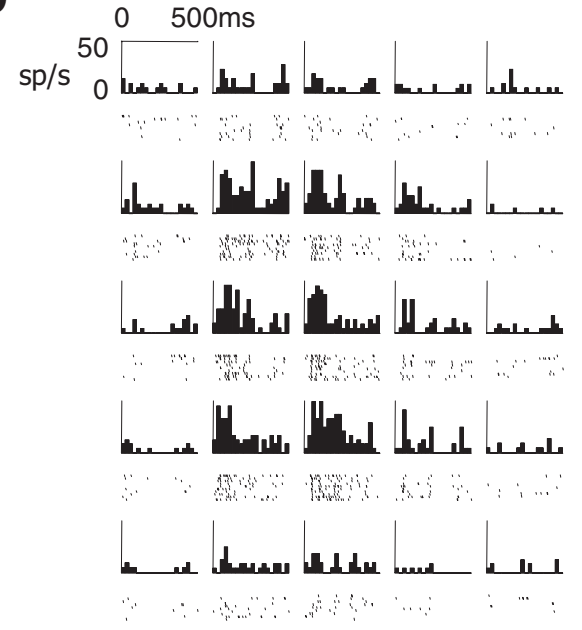

$\mathbf{F}$

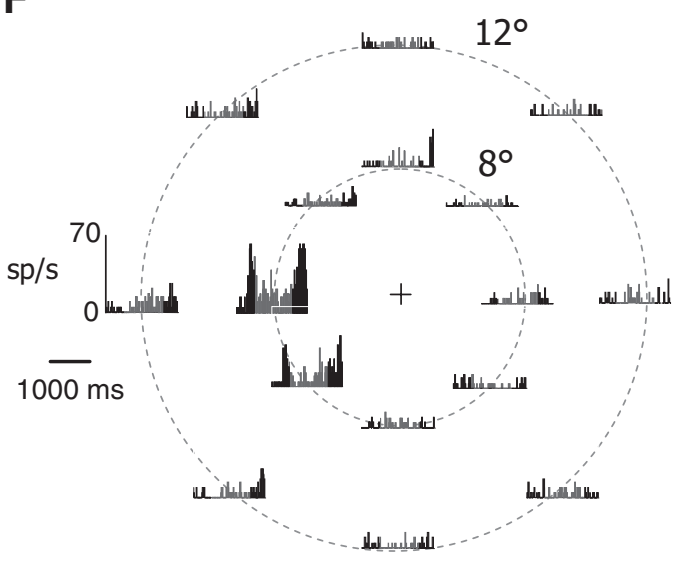

FIG. 1. $A$ and $B$ : illustration of the receptive field (RF) mapping procedure used to obtain the data in $C$ and $D$. Squares represent stimuli flashed at the positions of an imaginary matrix. Two matrices of different resolution were sequentially tested. The probed locations were spaced every $4^{\circ}$ for the $6 \times 6$ matrix $(A)$ and every $2^{\circ}$ for the $5 \times 5$ matrix $(B)$. The $5 \times 5$ matrix was centered at the location $\left(x=-6^{\circ} y=-2^{\circ}\right)$ that previously showed the strongest response from the coarser $6 \times 6$ mapping matrix. $C$ and $D$ : responses of 1 lateral intraparietal area (LIP) neuron to stimulation by the $6 \times 6$ matrix $(C)$ and $5 \times 5$ matrix $(D)$. Rasters and corresponding peristimulus time histograms show responses to the presentation of a flashed stimulus (200 ms) and to its disappearance (300 ms) for each location in the stimulation matrices. $E$ : smoothed RF map based on average responses from $D$. Dashed lines represent horizontal and vertical meridians. The cross is the fixation point. White dots indicate the 25 mapping locations that were probed. Black circle superimposed on the map represents the chosen position (center, $\left.x=-7^{\circ} y=-2^{\circ}\right)$ and relative size ( $4^{\circ}$ diameter) of the circular dot patch used in the main task for this particular cell. $F$ : responses of the same neuron during the memory delayed saccade task, run at 2 eccentricities. Each histogram shows the averaged binned response starting $250 \mathrm{~ms}$ before the onset of the flash to $350 \mathrm{~ms}$ after the extinction of the fixation point. Each histogram begins at the onset of the stimulus, and the gray shading indicates the 700-ms delay period between the offset of the stimulus and the extinction of the fixation point.

can be distinguished from neurons in adjacent areas based on their pattern of activity in this task, in particular their firing during the delay period (Barash et al. 1991; Cook and Maunsell 2002). On each trial, following fixation and a $700-\mathrm{ms}$ delay, a spot $\left(0.75^{\circ}\right.$ wide, $\left.64 \mathrm{~cd} / \mathrm{m}^{2}\right)$ was flashed for $200 \mathrm{~ms}$ at one of eight locations, $10-12^{\circ}$ from the fovea, separated by $45^{\circ}$ around the clockface. After a delay of 700 
(monkey $H$ ) or $1,000 \mathrm{~ms}$ (monkey $R$ ), the fixation point was extinguished, indicating that the monkey should make a saccade to the remembered location of the peripheral stimulus. The monkey was rewarded for initiating the saccade within $500 \mathrm{~ms}$ and maintaining eye position within $4^{\circ}$ of the position of the flashed spot for $\geq 250 \mathrm{~ms}$. Because we generally found an excellent correspondence between the response field of the neuron in the memory delayed saccade task and the RF-mapping task, for some cells we adjusted the eccentricity of the flashed spot to $5-8^{\circ}$ from the fovea if the RF mapping task showed a small RF closer to the fovea (e.g., Fig. $1 F$ ).

\section{Neuronal recording and data collection}

Recordings were made with commercial tungsten microelectrodes (75 $\mu \mathrm{m}$ diam, Frederick Haer), introduced into the cortex through a 26-guage stainless steel guide tube. A delrin grid was used to maintain the guide tubes in place in the recording cylinder and permitted reproducible electrode penetrations with a resolution of $1 \mathrm{~mm}$ (Crist et al. 1988). Before recording, we obtained a T1-weighted MRI scan to guide electrode penetrations. LIP was approached dorsally so that the medial and lateral banks of the intraparietal sulcus (IPS) were encountered sequentially. On most penetrations, we were able to distinguish a 1- to 2-mm "quiet zone" in which no single units were encountered between the medial and lateral banks of the IPS. We searched for units while the animal was performing the RF mapping task. Neuronal responsiveness to the RF mapping task increased dramatically once the electrode entered the lateral bank of the IPS. Once a cell was isolated in the lateral bank of the IPS and its RF mapped, we ran the main task one to three times depending on the number of speeds tested. We then ran the memory delayed saccade task, and if the unit was still well isolated, we also ran the single dot task.

\section{Assignment of neurons to cortical areas}

Neurons were localized to the lateral bank of the IPS based on the MRI. Neurons were considered to be in LIP if they had selective delay or saccade-related activity or were located between such cells in the same electrode penetration. While recording in the lateral bank of the IPS, we attempted to record the full sequence of tasks from any isolated unit that was at least minimally responsive in the RF mapping task. No other prescreening criteria were applied; in particular, we did not prescreen neurons based on their responses to motion stimuli. The few isolated neurons that did not meet this minimal criterion may have had RFs located beyond the edge of the stimulus monitor and therefore would presumably not have been stimulated effectively by the motion stimuli.

\section{Data analysis}

In the main task, average firing rates were computed over a 560-ms window starting $40 \mathrm{~ms}$ after the start of movement. An inspection of the data by eye showed that, for most of the cells, the onset of the motion response was well captured by this latency. In the single dot task, we typically averaged the firing rates over that part of the dot's trajectory that corresponded to the same region of space covered by the dot patch to facilitate comparison to the responses to the moving dot patch. Depending on the speed selected and the size of the dot patch used for the particular unit under study, the time window varied from $\sim 200$ to $500 \mathrm{~ms}$.

For each unit, the preferred direction and a measure of directionality [the direction index (DI)] were computed using a method adapted from circular statistics (Fisher 1993). Treating the average response to each direction as a vector, a mean vector was determined by computing the resultant and normalizing by a scalar equal to the sum of the average responses to each direction. We took the magnitude of the mean vector as the DI for the neuron and the angle of the mean vector as the preferred direction for the neuron. For a cell that is completely nonselective for direction (i.e., all directions of motion produce the same response), DI $=0$. For a cell that only responds to one direction of motion, DI $=1$.

A bootstrap permutation procedure was used to determine whether the DI of individuals neurons was different from that expected from chance. Trials from each neuron were randomly resampled without replacement (i.e., permuted) to form eight new sets of responses. The DI was computed, as described above, for the resampled data. This procedure was repeated 5,000 times. If the measured DI was $>99 \%$ of the resampled indices $(P<0.01)$, the null hypothesis that the cell was nondirectional was rejected. A Bonferroni correction was applied to the significance level because most of the neurons were tested with more than one speed. In subsequent analyses, for each neuron, we took the highest DI among the various speeds tested, which in our data set always corresponded to the DI that was least likely to have arisen by chance (i.e., lowest $P$ value in the bootstrap permutation test).

As a control for the DI calculation, for each neuron, we also computed a "direction index" from the visual response elicited by the onset of the stationary random dots patch. In this case, the set of responses to each direction of motion was taken as the set of responses to the stationary stimulus before the dots started moving. Because the response to the stationary dots should be the same regardless of the upcoming direction, the index calculated in this way should not be different from that expected from chance. The firing rates were averaged over a 250-ms window starting $40 \mathrm{~ms}$ after the onset of the stationary dots (for the few cells for which the dots were stationary for only $200 \mathrm{~ms}$ before moving, we averaged the firing rate over a 160 -ms window starting $40 \mathrm{~ms}$ after the onset of the dots). The "DIs" obtained in this fashion were subjected to the same bootstrap procedure described above.

For each cell, we constructed direction-tuning curves by fitting the averaged responses for each direction with a Gaussian function, using a nonlinear least squares optimization procedure that incorporated both the variance and the magnitude of the responses (McAdams and Maunsell 1999). The Gaussian function had the following form: $y_{\mathrm{i}}=a+b e^{-\left[\left(x_{\mathrm{i}}-c\right)^{2} / s^{2}\right]}$, where $y_{\mathrm{i}}$ is the cell's response to a stimulus moving in direction $x_{\mathrm{i}}$. Four parameters are free: $a$ is the minimum firing rate (asymptote), $b$ is the difference between the maximum and minimum firing rate (amplitude), $c$ is the preferred direction (mean), and $s$ is the SD (width) of the fitted Gaussian. The quality of fits was dependent on both the mean and the variability of the responses to each direction. The acceptability of each Gaussian fit was determined by performing an $F$ test $(P<$ 0.05 ) that compared the goodness of fit from the Gaussian with the goodness of fit obtained for a linear fit.

In the RESULTS, visual responses to the stimuli flashed in the RF mapping task are represented as gray-level two-dimensional maps. The RF maps were smoothed with a two-dimensional interpolation method using a best fitting third-degree polynomial function. In practice, 19 new points were generated between each two experimental data points (e.g., Fig. 1E). For some analyses, we estimated the center of the RF by averaging the $x$ and $y$ positions of all experimental and/or interpolated points in the map for which the corresponding response exceeded $1 / 20$ th the maximum response of the neuron. This estimate generally provided a good match to the center that one would assign by eye. We were also interested in asking how well the circular dot patch had been centered in the RF. For this we calculated a "visual gradient index" (VGI), which captured the differences in the strength of the smoothed RF map measured at the two intersections of the perimeter of the circular dot patch with any diameter crossing the dot patch. In particular, we were interested in knowing the VGI for the four axes of motion used in the experiment. From the large matrix made of interpolated points in the RF map, we derived the $x-y$ position and the corresponding average firing rate for each of the four axes of motion $\left(0-180,45-225,90-270\right.$, and $\left.135-315^{\circ}\right)$. For each of these 
axes, we calculated VGI as $\left(R_{\max }-R_{\min }\right) /\left(R_{\max }+R_{\min }\right)$. We designated the maximum of the four VGI values as max VGI.

\section{Analysis of fixational eye movements}

We analyzed eye movements made within the fixation window to examine whether they affected the firing of LIP cells. In particular, we were concerned that differences in fixational eye movements could affect neuronal responses in a way that mimicked direction selectivity. Horizontal and vertical eye positions were sampled every $5 \mathrm{~ms}$. We detected microsaccades in the eye position records using an adaptation of the procedure of Martinez-Conde et al. (2000). Eye position records were differentiated and smoothed with a $25-\mathrm{ms}$ sliding boxcar. The eyes were considered to be moving if the velocity was $>5 \%$ but not if any two successive velocity measurements differed in direction by $>30^{\circ}$. Movements that met these criteria were considered saccades if they were $\geq 10 \mathrm{~ms}$ in duration and $0.05^{\circ}$ in length and if there had not been a saccade in the previous $20 \mathrm{~ms}$. Microsaccades detected by this procedure agreed well with our qualitative assessment of microsaccades and adhered to the main sequence.

\section{RES ULTS}

\section{Basic responses of LIP neurons}

One hundred fifty-six neurons (80 from monkey $H$ and 76 from monkey $R$ ) were classified as being in LIP based on the anatomical and physiological criteria outlined in METHODS. These 156 units provided the data that are analyzed here.

The RF mapping procedure was highly effective in delineating the RF location for the vast majority of LIP neurons. For example, the mapping procedure with the $6 \times 6$ stimulus matrix elicited a spatially focal response $\sim 6^{\circ}$ from the fovea for the neuron shown in Fig. $1 C$. We used the finer $5 \times 5$ stimulus matrix to map the neuron's RF with higher spatial resolution (Fig. 1D). The circle on the smoothed high-resolution RF map in Fig. $1 E$ indicates the location and size of the circular dot patch used in the main task for this neuron. The responses of the same neuron during the memory delayed saccade task are shown in Fig. $1 F$. The neuron showed strong visual, delay, and saccade-related activity, and the location of the response field shown by this task closely corresponded to the location of the RF shown by the RF mapping task. Responses to the memory delayed saccade task will be examined in more detail later.

\section{Responses of LIP neurons to the random dot motion patches}

Many LIP neurons responded in a direction-selective manner to the random dot motion stimulus. Figure $2, A$ and $B$, shows the responses of two different LIP units to the onset of the dot patch and the motion of the dots in eight different directions. The two neurons were clearly direction selective, responding best to motion in one direction (preferred direction) than the opposite (null) direction. As in other direction-selective visual areas, we found that the degree of direction selectivity could vary considerably among LIP neurons. For the two neurons in Fig. 2, $A$ and $B$, for example, the ratio of the preferred-direction response to the null-direction response was $2: 1$ and $1.7: 1$, respectively.

The directionality of each unit was quantified by measuring a DI, which is the normalized vector sum of the responses to each direction (see METHODS). A completely selective cell responding only to motion in one direction would have a DI =
1, whereas a completely nonselective cell responding in the same way to all directions would have a DI $=0$. The cells in Fig. 2, $A$ and $B$, have DIs $=0.15$ and 0.2 , respectively.

The distribution of the direction indices for all 156 LIP neurons is shown in Fig. $3 A$. Sixty-one percent of the units $(95 / 156)$ had a direction selectivity greater than expected from chance (Fig. 3A, black bins), using the bootstrap permutation test described in METHODS. The median DI of all 156 cells was 0.09 , and the median DI of the 95 units with a statistically significant DI was 0.13 . For the bootstrap permutation test, we computed a distribution of DIs from randomly permuted data for each unit, and we computed the mean DI of these permuted values for each unit. The distribution of those mean DIs (expected from chance) is shown for all units in Fig. $3 B$ and is clearly shifted toward lower DIs compared with the distribution of actual DIs in Fig. $3 A$ (median $=0.03$ ).

As a control for our analysis method, we also calculated a distribution of "direction" indices from the visual response of each cell to the onset of the random dot patch before the dots started moving. Because the visual responses to these stationary dots should be the same irrespective of the upcoming direction, the DIs should be close to 0 - which we indeed found (median, 0.03; Fig. 3C). We also performed the bootstrap permutation test on the responses to the stationary dot patches, and no single unit (0/156) showed a DI greater than that expected from chance. These negative results suggest that our method for calculating a DI was not prone to incorrectly classifying nondirectional responses as directional.

To give a more direct measure of the magnitude of the difference in response between preferred and null directions for each neuron, we also calculated a normalized response ratio between preferred and null directions according to the formula: [1 - (response to null direction/response to preferred direction)]. The normalized response ratio ranges from 0 to 1 , and a cell with a 2:1 ratio between preferred and null responses would have a normalized response ratio of 0.5 . The median normalized response ratio for all 156 LIP units was 0.3 , and the median for the 95 significantly direction-selective units was 0.4 , indicating that, for one half of these 95 units, the preferreddirection response was $\geq 1.7$ times greater than the nulldirection response. In the rest of this paper, we will refer to this subpopulation of 95 LIP cells with statistically significant DI as the direction-selective cells.

\section{Direction tuning of LIP neurons}

To look more closely at the direction tuning in LIP neurons, we constructed a tuning curve for each cell by fitting a Gaussian function to the cell's average responses as a function of direction. Direction-tuning curves for neurons in other visual cortical areas, such as MT, can be well fit with a Gaussian (Albright 1984; Snowden et al. 1992). We fit direction-tuning curves only for the subpopulation of 95 direction-selective LIP neurons. For 60/95 (63\%) of those neurons, the Gaussian provided a statistically significant fit to the tuning curve using the goodness-of-fit criterion described in METHODS ( $F$ test, $P<0.05$ ). Fitted tuning curves are shown for two individual LIP units in Fig. $4, A$ and $B$. Figure $4 C$ shows the average direction-tuning curve for the entire population of 95 direction-selective LIP neurons, obtained by aligning the preferred response of each cell on $0^{\circ}$ and averaging the re- 

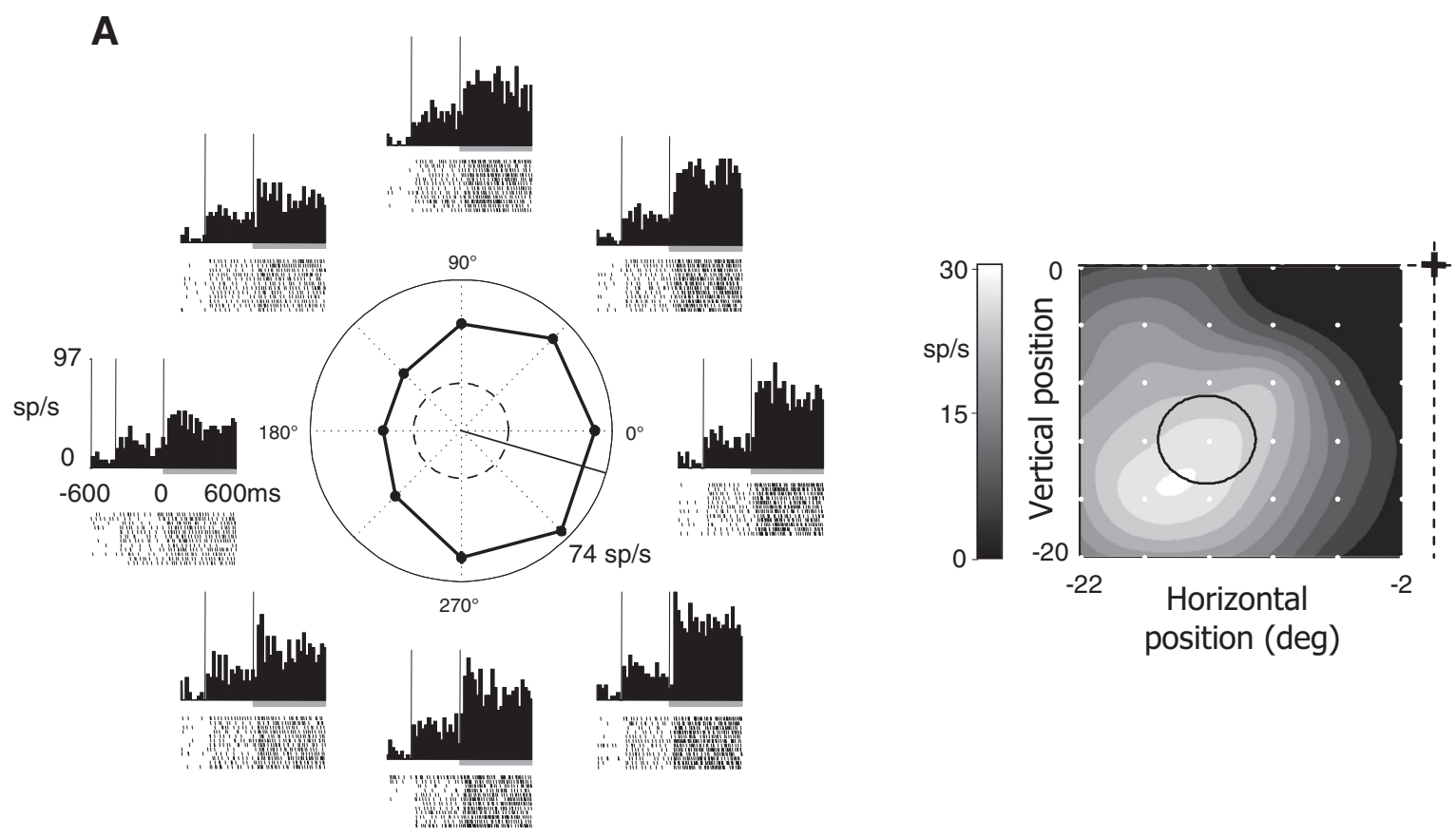

B
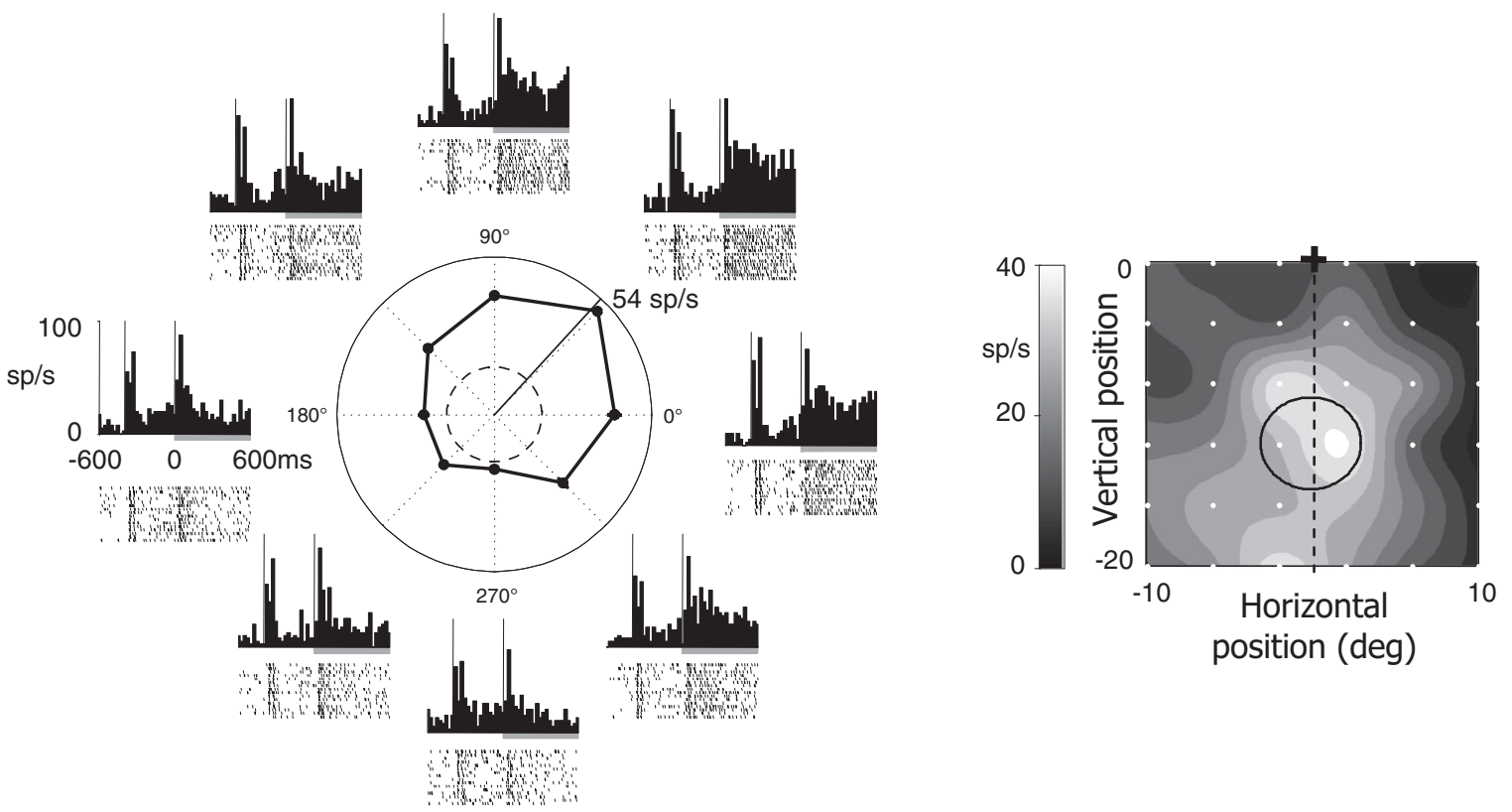

FIG. 2. $A$ and $B$ : direction selectivity of 2 LIP neurons. Histograms show responses to 8 different directions starting $200 \mathrm{~ms}$ from the presentation of the random dot patch. Vertical lines indicate the appearance of the stimulus and the start of movement, respectively, and the horizontal gray bar indicates the period of movement. The polar plot in the center shows the averaged response for each direction over a 540-ms window starting $40 \mathrm{~ms}$ from the start of movement. The dashed circle shows the average firing rate to the stationary dots over a 200-ms window before motion onset. The radial line indicates the preferred direction, as determined from the mean vector. Bars indicate SE. For these 2 cells, the direction index (DI) was $0.15(A)$ and $0.20(B)$. RF maps to right are as described in Fig. 1. Black circles indicate placement of the circular dot patch.

sponses across cells. For the remaining 35/95 neurons for which the Gaussian did not provide a statistically significant fit to the tuning curves, the curves generally still appeared unimodal and continuous, and we did not find evidence for more complex direction tuning, such as bimodal tuning curves.

To quantify the sharpness of direction tuning for the 60 cells that were well fit by a Gaussian, we took the full width at half height of the fitted Gaussian as a measure of the width of the tuning curve. Tuning width varied considerably among individual neurons. The two cells in Fig. 4, $A$ and $B$, have tuning widths of 150 and $130^{\circ}$, respectively. The distribution of tuning width for all 60 neurons that were well fit with a Gaussian is shown in Fig. 4D. The median tuning width for this subpopulation of neurons was $136^{\circ}$. 
A

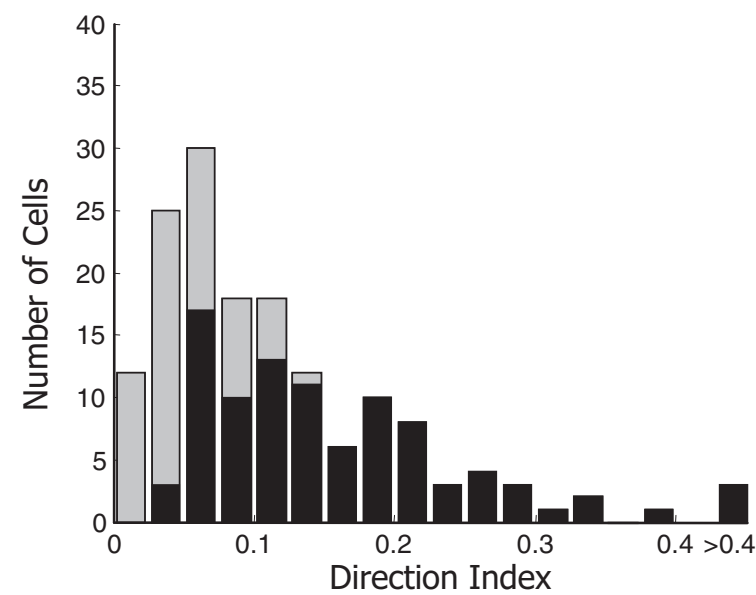

B

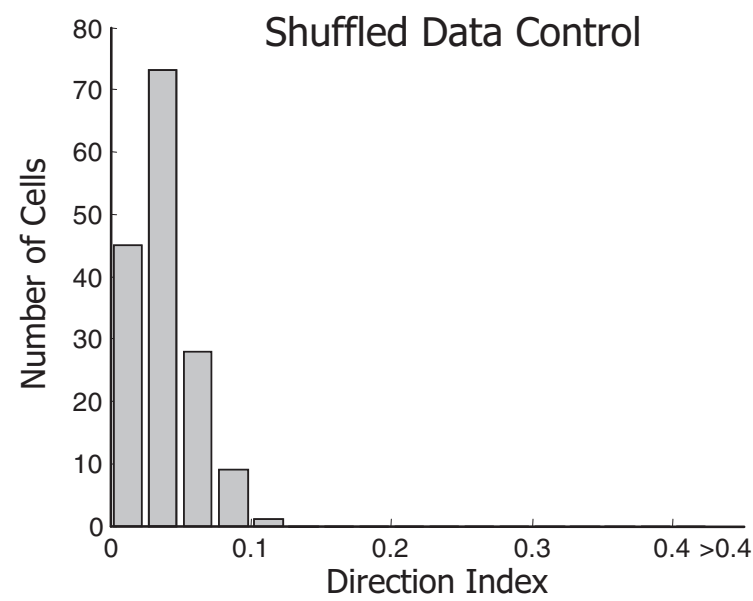

C

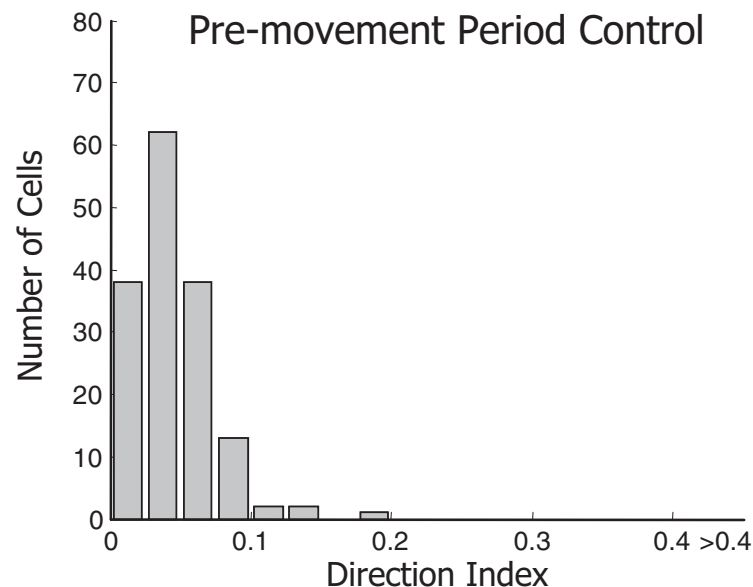

FIG. 3. A: distribution of direction indices for the entire population of LIP neurons $(n=156)$. Black bars indicate the 95 units showing a significant DI $(P<0.01)$. B: distribution of DI expected from chance for the same population, derived using a bootstrap permutation. $C$ : distribution of DIs computed from the visual response elicited by the onset of the stationary random dots patch for the same population.

The Gaussian was fitted by varying four parameters: amplitude, SD, asymptote, and preferred direction. An interesting finding was that the asymptote was generally higher than the "baseline" activity measured during the period when the animal fixated before the dot patch was turned on. For example, the asymptotic level for the population-tuning curve in Fig. $4 C$ was $\sim 20$ spikes/s higher than the average baseline activity (heavy dashed line in Fig. 4C). However, the asymptotic level was very close to the average response of the cells to the stationary dots measured during the final $200 \mathrm{~ms}$ before the dots began moving (fine dashed line in Fig. $4 \mathrm{C}$ ), indicating that the presence of the stationary dots in the receptive field caused a tonic increase in activity well above the baseline level of activity. This can be most clearly seen in Fig. 5A, which shows the average responses across all LIP neurons as a function of time (excluding the 34 neurons for which we used a shorter premotion period). If we focus on the direction-selective neurons, once the motion began, the average response increased for motion in the preferred direction and (following a transient increase) decreased for motion in the null direction compared with the tonic level of firing in response to the stationary dots (Fig. 5B). Thus the direction-selective response to the moving dots appears to be superimposed on a static offset in activity elicited by the presence of the stationary dots in the RF. In this view, the static offset would tend to reduce the direction selectivity of LIP neurons. To illustrate this point, for the 82/95 direction-selective LIP neurons included in Fig. $5 B$ (excluding the 13/95 direction-selective cells for which we used a shorter premotion period), we recalculated the normalized response ratio [1 - (null response/preferred response)] after first subtracting the "baseline" activity averaged over the 200-ms period before the dots began moving. With this procedure, the mean normalized response ratio was 0.73 for LIP.

\section{Time course of direction selectivity}

In addition to the nonspecific static offset in activity, there was also a prominent transient in activity at the onset of motion that was similar in amplitude for all directions (Fig. 5B). This suggests that the responses are less direction selective immediately following the onset of motion. For our 95 directionselective LIP neurons, we recalculated the direction index in two consecutive $250-\mathrm{ms}$ periods: $40-290 \mathrm{~ms}$ following the start of movement and 290-540 ms following the start of movement. The average DI was indeed significantly higher for the later time period: $0.18 \pm 0.01$ versus $0.15 \pm 0.01$ (SE; 2 -tailed $t$-test; $P<0.05$ ). To examine the time course in more detail, we computed a running average of the direction index (Fig. 5C). For each unit, we calculated the DI every $50 \mathrm{~ms}$, averaging responses to each direction over the 100-ms interval centered on each 50-ms time point, and we averaged the time course among all 82 units included in the analysis. The average DI was elevated above baseline within $50 \mathrm{~ms}$ following motion onset, about halfway to peak by $100 \mathrm{~ms}$, and at peak by $\sim 150-200$ ms (Fig. 5C).

\section{Distribution of preferred directions in LIP}

The normalized vector-sum analysis provided a preferred direction for each neuron in addition to a direction index. The preferred direction for each of the 95 direction-selective LIP neurons is indicated by an arrow in Fig. 6 (filled arrowheads for monkey $H$ and open arrowheads for monkey $R$ ). The origin of each arrow was placed at retinotopic coordinates corresponding to the center of the dot patch used for that neuron (which for each neuron was positioned at or near the center of the neuron's RF; see METHODS). Although preferred directions were broadly distributed in all directions, there appeared to be 
A

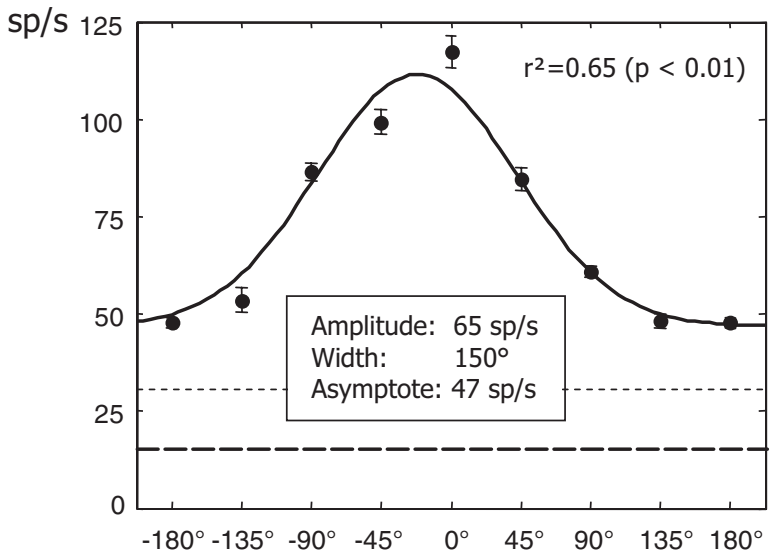

Motion direction relative to preferred direction

C

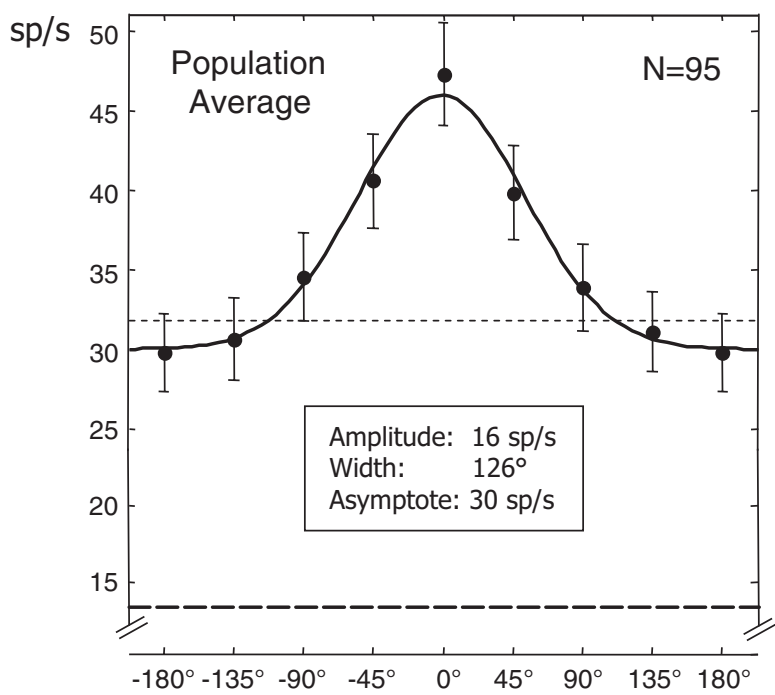

Motion direction relative to preferred direction
B

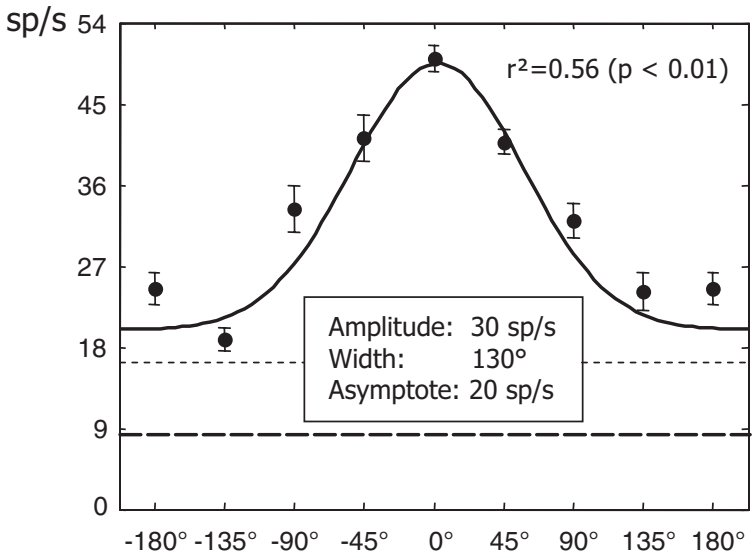

Motion direction relative to preferred direction

D

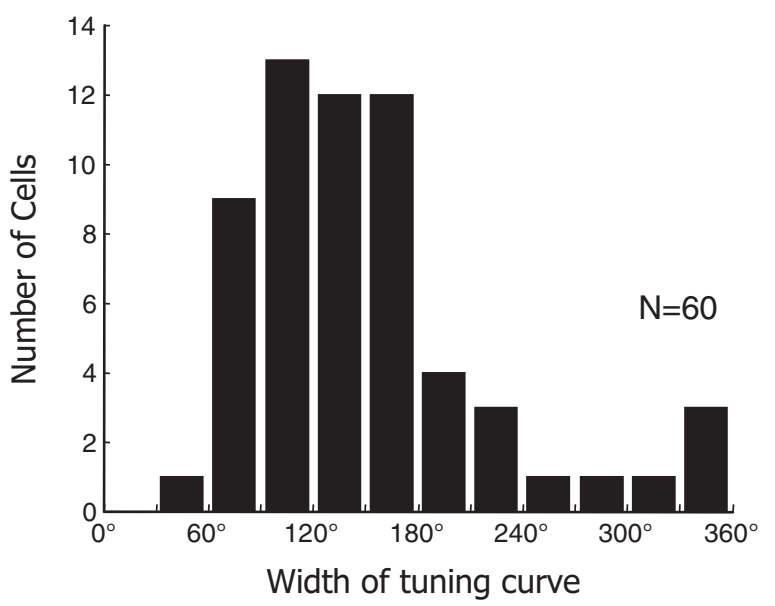

FIG. 4. $A$ and $B$ : direction-tuning curves for 2 LIP neurons (the neuron in $B$ is the same as that in Fig. $2 B$ ). The goodness of fit for each unit is indicated by

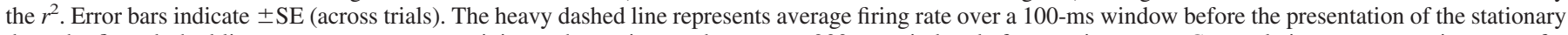

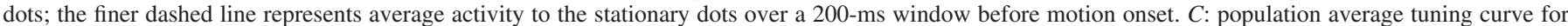

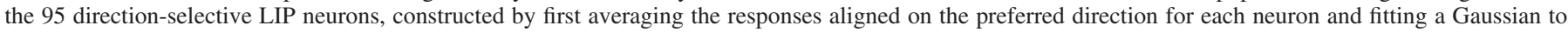

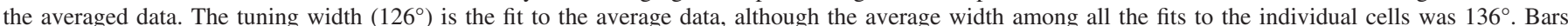

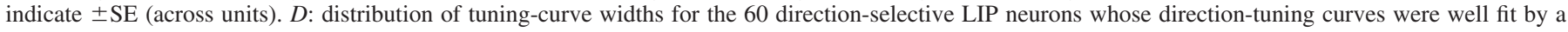
Gaussian.

a general bias for preferred directions toward the center of the visual field. If we replotted each neuron's preferred direction relative to the fovea (by projecting a unit vector in the neuron's preferred direction onto a line intersecting the fovea and the center of the neuron's RF), we found that for both animals there was a significant bias toward the fovea versus away from the fovea (Rayleigh test, $P<0.05$ for monkey $H$ and $P<0.001$ for monkey $R$ ). We also looked for a bias in preferred directions toward or away from the vertical meridian by projecting each preferred direction along the horizontal axis and found a bias toward the vertical meridian (Rayleigh test, $P \leq 0.001$ for both animals). However, we also found a statistically significant bias toward the horizontal meridian, at least for one animal
(Rayleigh test, $P>0.05$ for monkey $H$ and $P<0.001$ for monkey $R$ ). Thus while preferred directions are broadly distributed in LIP, across the population there may be a bias in preferred directions generally toward the center of the visual field, with at least some component of bias toward the fovea.

\section{Responses of LIP neurons to a single moving spot}

Some direction-selective neurons in other visual cortical areas have been shown to respond differently to a solitary moving spot than to a patch of moving dots. This property may be important for distinguishing solitary visual targets from background motion during visually guided movements (Kom- 
A

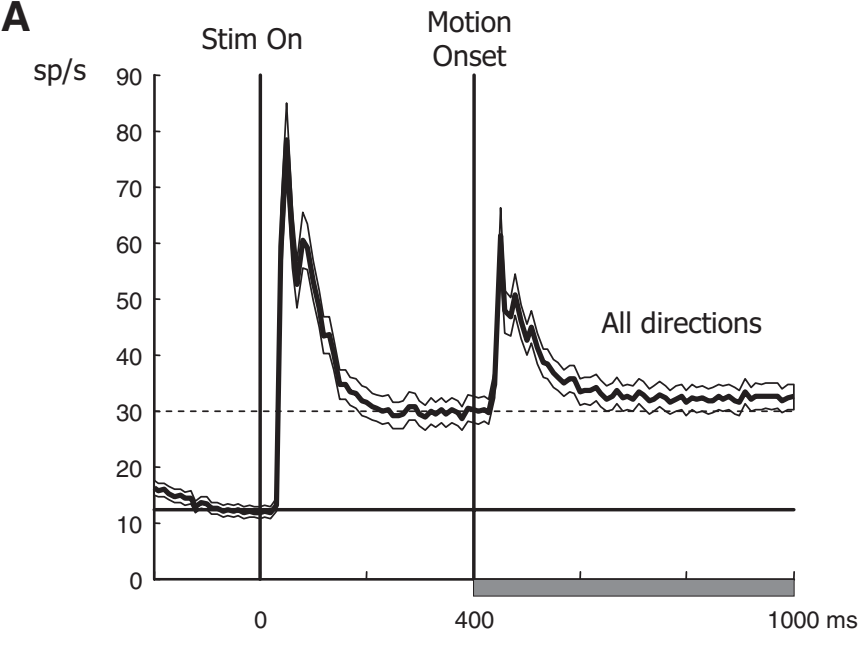

B

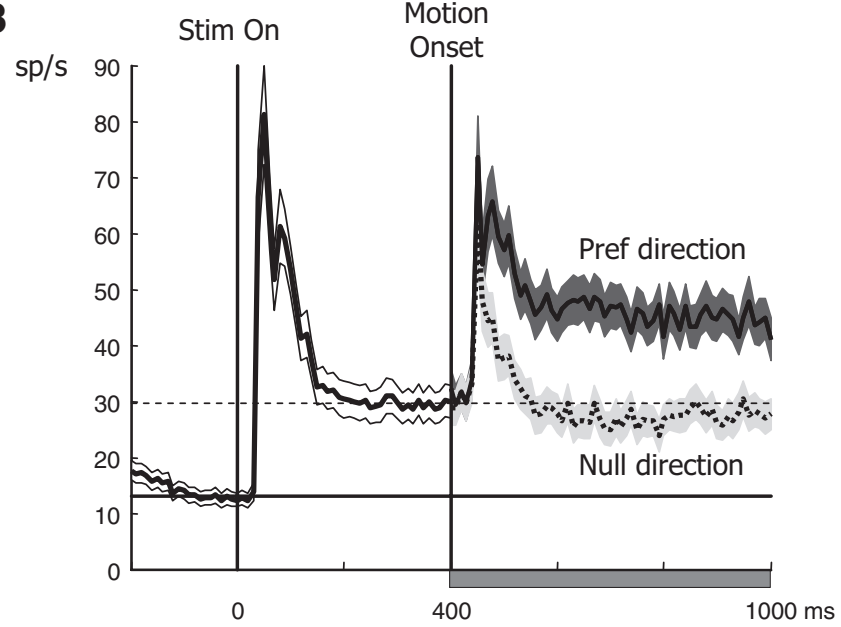

C

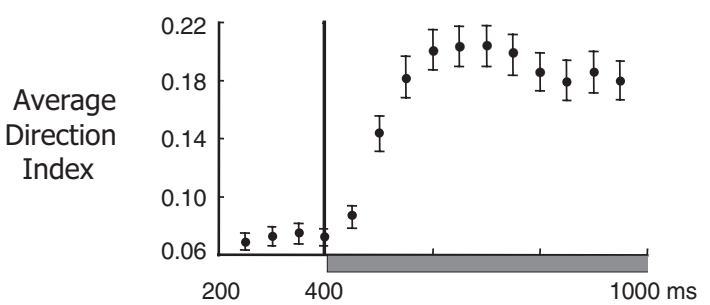

FIG. 5. Population-average activity for $(A)$ all LIP neurons with a 400-ms delay before motion onset $(n=122)$ and for $(B)$ the direction-selective neurons from that same set $(n=82)$. (Thirty-four neurons were excluded from the plots because the duration of the stationary dots for those units was $<400 \mathrm{~ms}$.) Responses start $200 \mathrm{~ms}$ before the presentation of the dot patch and extend until the end of the 600-ms motion period. In $B$, the average activity is shown separately for the preferred direction (continuous line) and null direction (dotted line). Transparent and shaded gray areas indicate \pm SE (across units). Horizontal gray bar indicates the motion period. $C$ : time course of the DI, averaged among the same 82 direction-selective neurons as in $B$. Bars indicate $\pm \mathrm{SE}$ (across units). Time base is the same as in $A$ and $B$. Note that the average DI before movement onset was slightly more elevated than that in Fig. $3 C$. This is because the shorter averaging interval $(100 \mathrm{~ms})$ results in a noisier estimate of the DI, and the DI can only take on positive values. In $A-C$, the horizontal gray bars along the time axis indicate the period in which the visual stimulus was moving.

atsu and Wurtz 1988). We thus examined whether LIP neurons also show this distinction. Of the 156 LIP cells in our study, 85 were tested with a single moving spot in addition to the random dot-patch motion stimulus (see METHODS). Our goal was to examine whether there were systematic differences in direction selectivity or responsivity between the two tasks for separate populations of cells, such as broad anticorrelations in directionality or responsivity between the two tasks across the population of cells.

Figure 7, $A$ and $B$, shows responses and direction-tuning curves for the single dot task for the same two LIP neurons that were shown in Fig. 2. Both neurons also responded well to the single moving spot, and there was a good correspondence between the direction-tuning curves for the two types of stimuli. To describe motion responses to the two types of stimuli, for each of the 85 neurons we compared the DI and the preferred direction. The median DI values were comparable between the dot-patch and single spot stimuli (Fig. $7 C$; medians, 0.09 and 0.07 , respectively) and were also strongly positively correlated rather than anticorrelated (correlation test, $r=$ 0.66, $P<0.001$; Fig. $7 C$ ). There were, however, a small number of neurons that were considerably more direction selective in one task than the other (Fig. 7C).

The statistical significance of the DI for the single spot stimulus was assessed using the bootstrap permutation test, and $50 / 85(59 \%)$ neurons had a significant DI $(P<0.01)$. Among the same 85 cells, $55 / 85(65 \%)$ had a significant DI for the dot-patch stimulus, and 41/85 (48\%) cells had a significant DI for both types of stimuli (black circles in Fig. 7C). Twentyseven percent (23/85) of the cells had a significant DI in only one of the two tasks-however, assuming independence, far more of the cells (47\%) should have been significant in only one task. This immediately argues against the strong hypothesis that the directional neurons are divided into distinct subpopulations favoring one or the other stimulus type.

For the 41 cells that showed significant directionality for both stimuli, the median DI values were comparable: 0.14 and 0.13 for the main task and the single dot task, respectively. For the 41 cells that showed significant directionality for both stimuli, we calculated the difference between preferred directions for the two types of stimuli. While there was some scatter, more than one half of the cells showed a difference in preferred direction $<45^{\circ}$ (median difference: $36^{\circ}$ ), which is the limit of

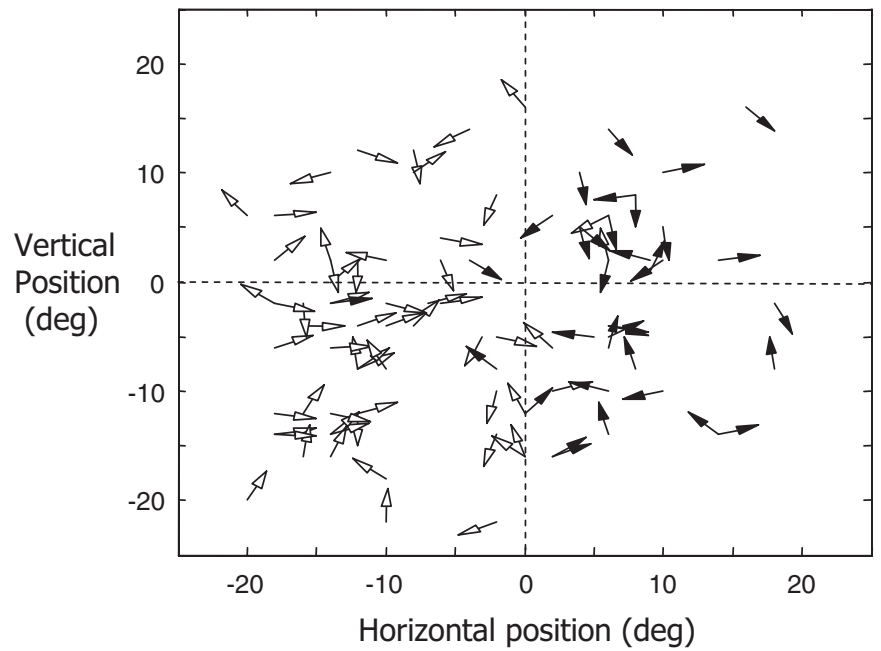

FIG. 6. Distribution of preferred directions for all 95 direction-selective LIP neurons. The origin of each arrow corresponds to the retinotopic coordinates of the center of the RF for 1 neuron, and the direction of the arrow corresponds to the preferred direction. Filled and open arrowheads indicate neurons for monkeys $H$ and $R$, respectively. 
A

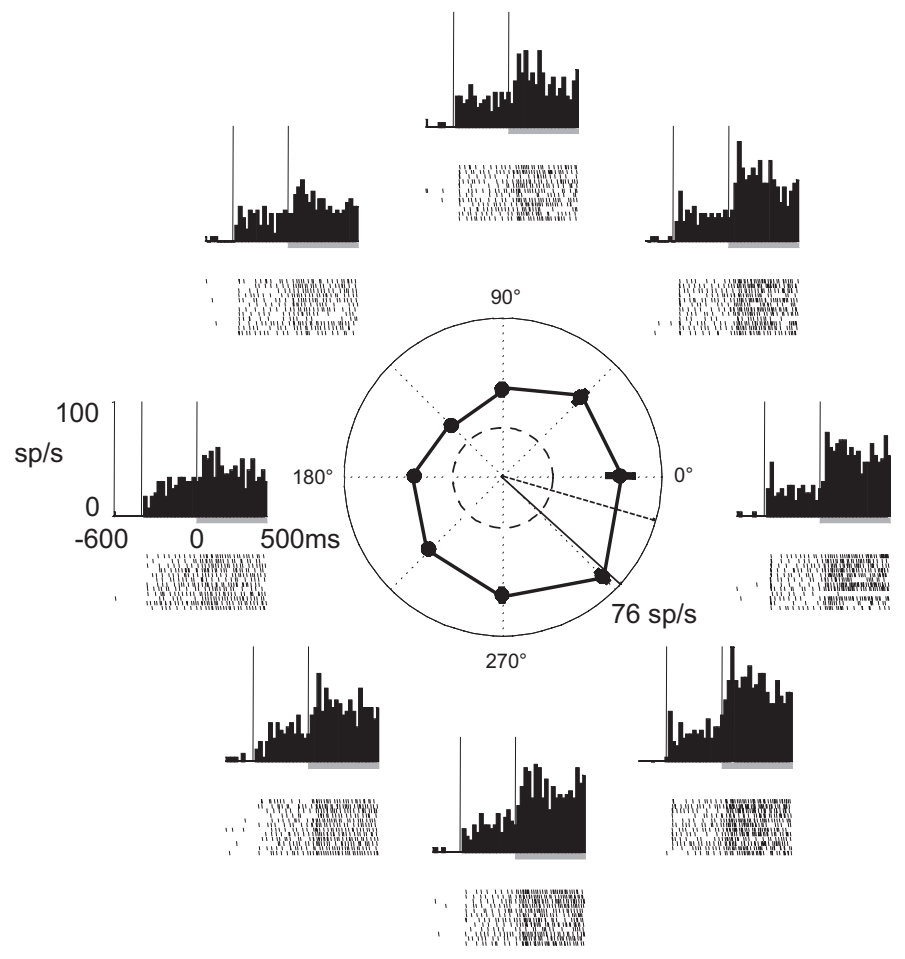

C

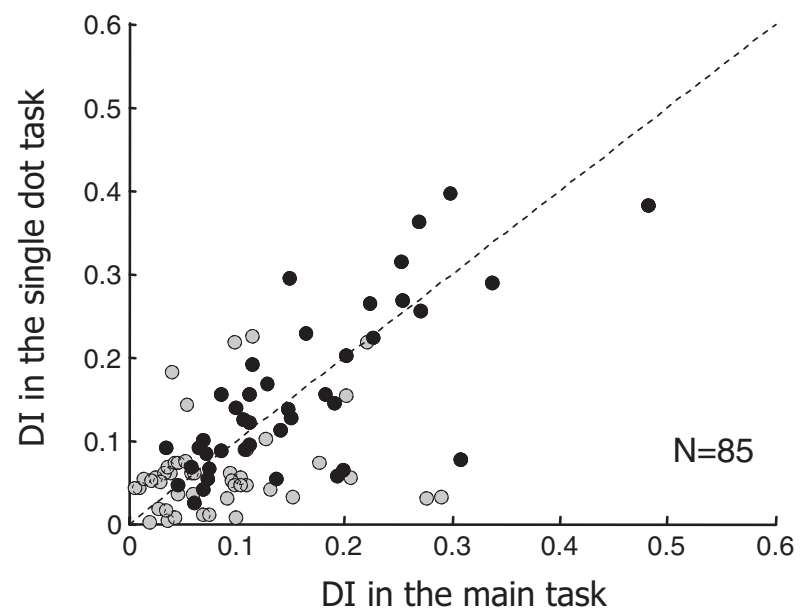

B
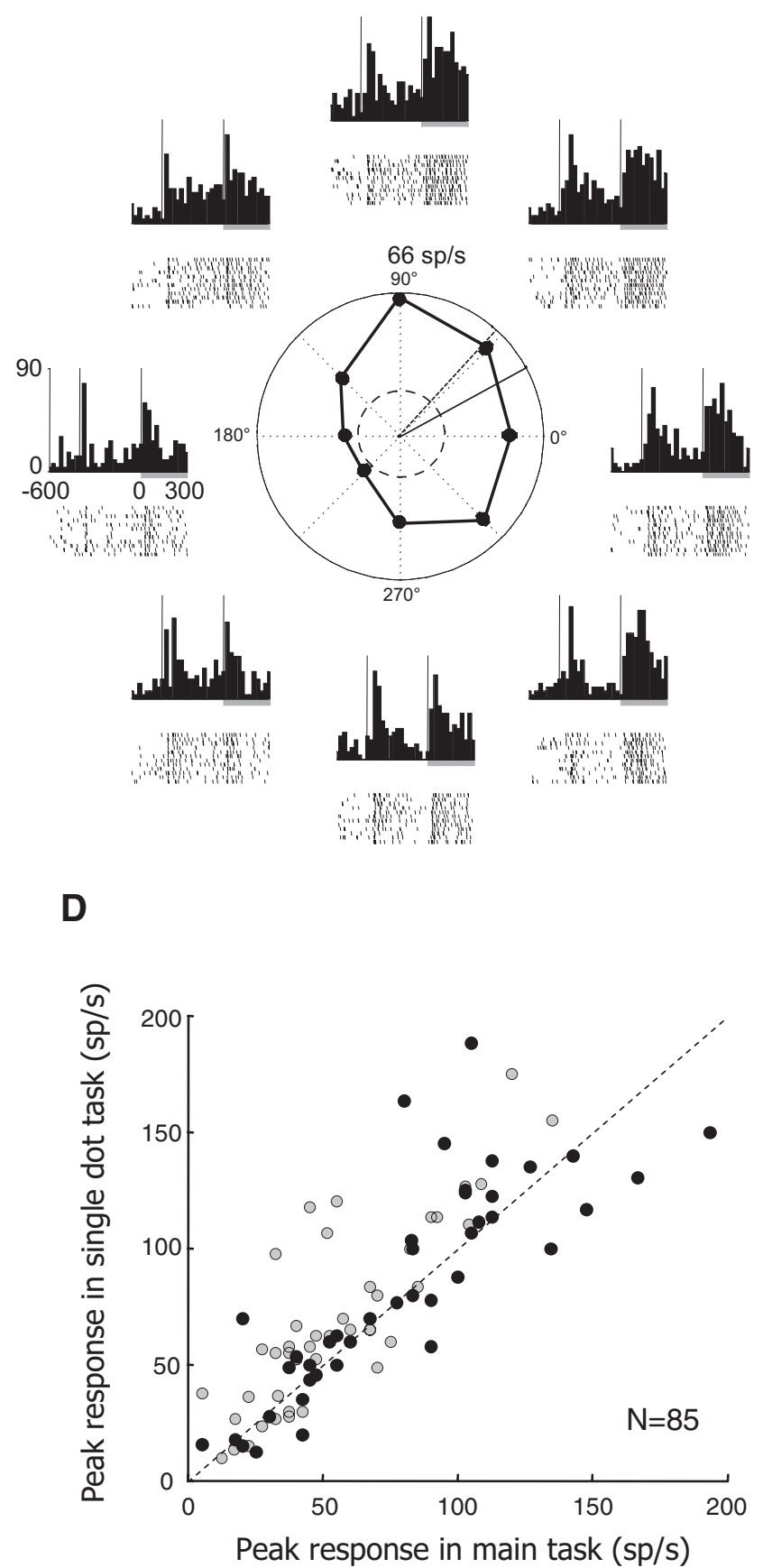

FIG. 7. $A$ and $B$ : responses and direction tuning in the single dot task for the same 2 LIP units that were shown in Fig. $2, A$ and $B$. Same conventions as in Fig. 2. For each unit, the solid radial line on the polar direction-tuning curve indicates the preferred direction for the single dot task, as determined from the mean vector. The dashed radial line indicates the preferred direction from the dot-patch task for the same unit for comparison. For the cell in $A$, the DI was 0.14 for the single dot and 0.15 for the dot patch; for the cell in $B$, the DI was 0.20 for both motion stimuli. $C$ : DI in the main task plotted as a function of the DI in the single dot task for the 85 LIP neurons that were tested with both tasks. $D$ : peak response amplitude to motion in the preferred direction for the main task vs. the single dot task. In both $C$ and $D$, black circles represent the 41 cells that showed significant directionality for both tasks, and the dashed lines have a slope of 1 .

our directional sampling. However, nine neurons had differences in preferred direction $>90^{\circ}$.

We also compared each of the 85 LIP neuron's peak response amplitude to the preferred direction of motion for both types of stimuli (Fig. 7D). The median peak response was slightly larger for the single spot stimulus ( 65 spikes/s) than the dot-patch stimulus (55 spikes/s), but this not unexpected. For example, for some units the single spot may have moved into a stronger part of the RF at the start of motion, whereas the dot patch always subtended the same part of visual space. However, the peak responses were not anticorrelated between the two types of stimuli but rather strongly positively correlated 
(correlation test, $r=0.82, P<0.001$ ). Thus overall, we did not find evidence that the bulk of directional LIP neurons are specialized for signaling one type of motion or the other. Nonetheless, there was a subset of cells that was considerably more directional in one task than the other or that had large differences in preferred direction between the two tasks. This could warrant further study.

\section{Responses of LIP neurons in the memory delayed saccade task}

Overall, our results indicate that many LIP neurons show reliable direction selectivity and that some LIP neurons can be strongly direction selective. Direction selectivity is not usually attributed to LIP neurons. Rather, many previous studies have shown that LIP neurons encode static spatial locations-the presence of visual stimuli within the response field and attention and/or planned eye movements to the response field. These responses are commonly shown using a memory delayed saccade task, in which the location of a flashed visual target must be remembered during a delay period before the animal makes a saccade to the target's former location. The memory delayed saccade task shows a distinct pattern of activity in LIP, with many neurons continuing to be active during the memory delay and/or increasing their activity just before the saccade is initiated (Andersen et al. 1985; Barash et al. 1991). This was also true for the LIP neurons in our study (or, more properly, we took the responses in the memory delayed saccade task as one of our criteria for identifying LIP neurons; see METHODS). Figure $8 \mathrm{~A}$ shows population-average responses among the 143/156 LIP neurons in our study that were tested with the memory delayed saccade task. On average, LIP neurons showed strong, sustained activity during the delay period, with a slight increase in firing beginning several hundred milliseconds before the saccade onset.

It is possible that the direction-selective neurons that we have found in LIP constitute a distinct population within LIP that may not have been examined in previous studies. If so, the direction-selective neurons may respond differently in the memory delayed saccade task. Figure $8, B$ and $C$, shows the population average responses in the memory delayed saccade task for just direction-selective neurons (Fig. $8 B$ ) and for the remaining, nondirection-selective neurons (Fig. $8 C$ ). Both sets of neurons had robust sustained firing during the delay period, although correlation analysis showed a weak but significant trend for both the delay activity and the visual response to be smaller for neurons with larger direction indices (delay activity: $r=-0.22$; responses averaged from 150-650 ms following stimulus offset; visual response: $r=$ -0.27 , responses averaged from $40-200 \mathrm{~ms}$ after stimulus onset, measured at the location which elicited the largest response; $P<0.01$ in both cases).

For each neuron, we calculated a spatial selectivity index for the delay activity and presaccadic activity using the same method that we used to calculate the DI (the normalized mean vector; see METHODS). There was no detectable relationship between DI and the selectivity index for delay activity (Fig. $9 A$ ) or presaccadic activity (Fig. $9 B$; correlation analysis, $r=0.17, P>0.1$, and $r=0.18, P>0.05$, respectively). We also averaged the responses in the memory delayed saccade task of the seven LIP units with the highest DI values (all $>0.3$ ), and their average response was similar to that of the entire population of LIP neurons (Fig. 9C). Thus while there may be subtle differences between the responses of directional and nondirectional neurons to the memory delay saccade task, the directional neurons nonetheless had robust delay activity. Importantly, this suggests that our finding directional neurons was not caused by straying into the neighboring ventral intraparietal area (VIP). VIP contains a preponderance of direction-selective neurons (Colby et al. 1993; Zhang et al. 2004), but VIP neurons show very little sustained delay activity in the memory delayed saccade task (Cook and Maunsell 2002).

\section{Fixational eye movements and direction selectivity}

In response to different directions of motion in the RF, the animals might make different eye movements within the fixation widow, and these eye movements might in turn affect the firing of LIP neurons in a way that mimicked direction selectivity. We thus examined microsacadic eye movements, using an analytical procedure modified from Martinez-Conde et al. (2000) (see METHODS). With the 600-ms stimulus presentations that we used, on most presentations, we detected zero, one, or two microsaccades per presentation. This allowed us to make a worst-case comparison- between presentations with zero microsaccades and presentations with at least one microsaccade. For all cells for which we had at least one presentation with zero microsaccades for each of the eight directions $(n=70$ cells), we calculated two new direction indices: one for the stimulus presentations with microsaccades and one for the presentations without microsaccades. If the pattern of microsaccades was responsible for creating an artifactual "direction selectivity," we should have found that the "with microsaccades" DI was different from the "without microsaccades" DI. Instead, we found that the two DI measures were very similar: across all 70 neurons, the median DI for without microsaccades and with microsaccades was 0.14 and 0.13 , respectively, and the two DI measures were strongly positively correlated $(r=$ $0.80 ; P<0.001)$. Thus the direction selectivity in LIP was not an artifact of fixational eye movements.

\section{Distinguishing direction selectivity from static spatial encoding}

The direction selectivity in LIP appears to be a signal that is distinct from the well-known property that LIP neurons encode spatial salience and/or plan to saccade to static locations. There are situations, however, in which static spatial encoding could masquerade as direction selectivity. For example, if a single spot of light moves into the RF from one direction and out of the RF in the opposite direction, differences in firing between the two directions could be misinterpreted as direction selectivity. This scenario cannot explain the direction selectivity in our experiment, because we used a patch of moving dots that always subtended the same part of visual space for all directions of motion. However, although our animals had no task except to fixate, it is possible that they may have reflexively and covertly attended to different spatial locations depending on the direction of the dots within the patch. A hypothetical worst case might be if opposite directions of motion within the dot patch attracted the animal's attention to opposite sides along the dot-patch's perimeter. For example, the animals' 

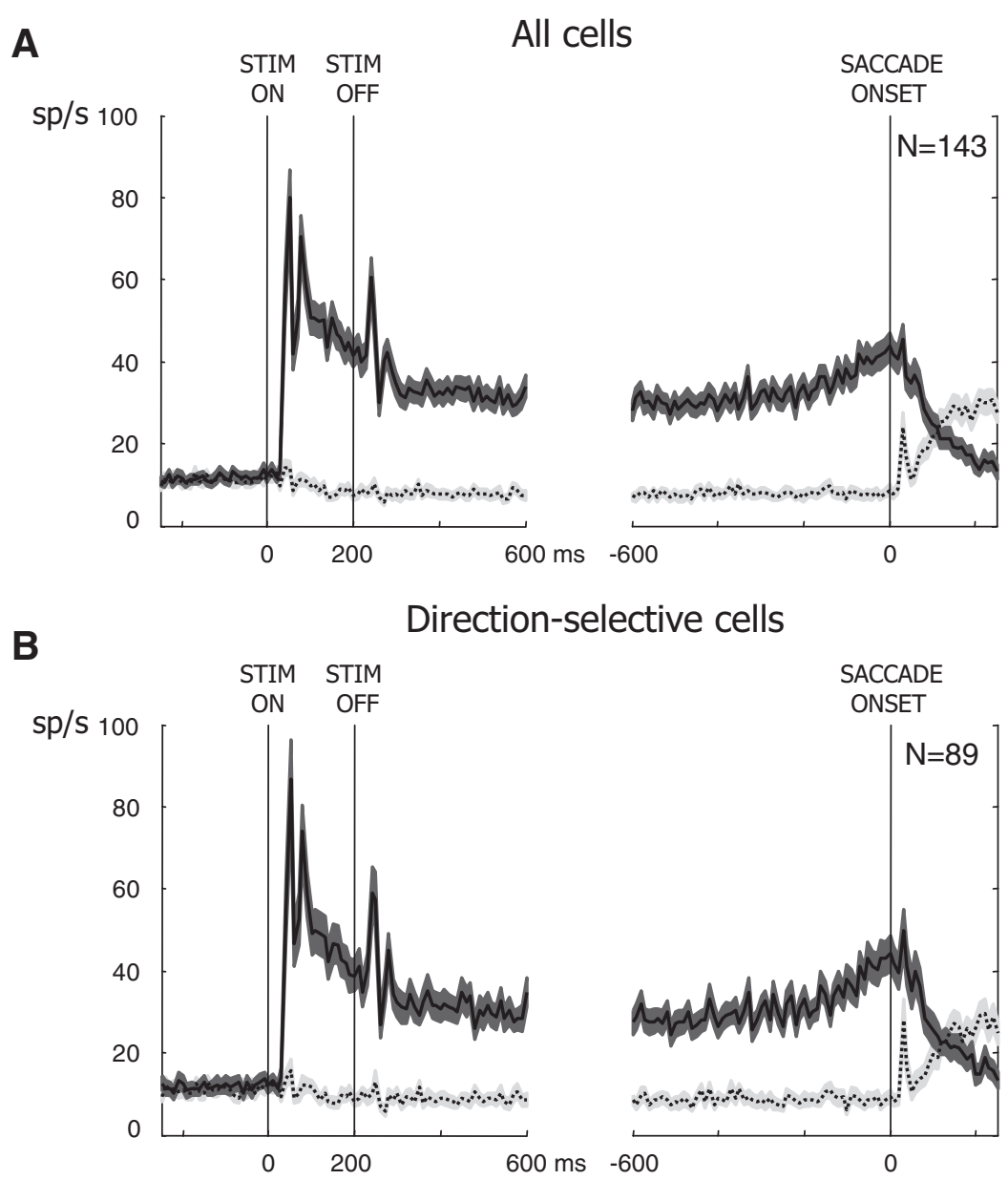

FIG. 8. A: population-average response to the memory delayed saccade task for all 143 LIP cells tested with that task. The plot on the left is aligned to stimulus appearance, and the plot to the right is aligned to saccade onset. The continuous line indicates the response to the preferred location, and the dotted line indicates the response to the opposite location. Superimposed shaded areas along the response profiles represent \pm SE. $B$ and $C$ : population-average responses to the memory delayed saccade task for direction-selective cells $(n=89)$ and nondirection-selective cells $(n=54)$, respectively.

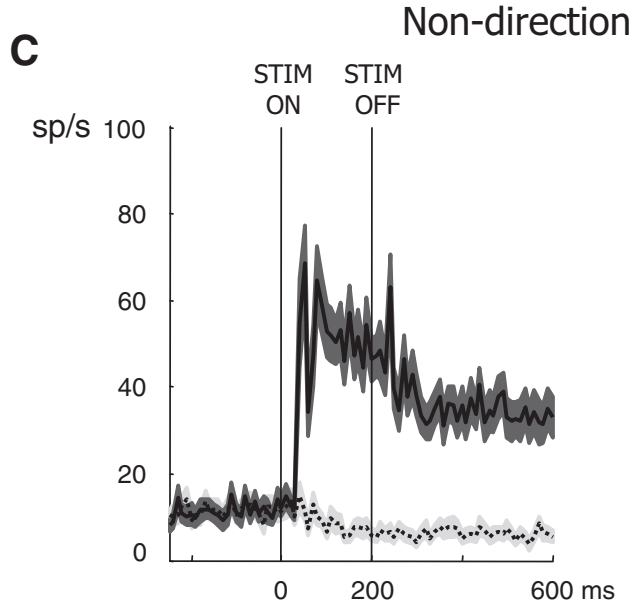

attention may have been drawn to the side of the patch where the dots appeared to be "headed" or to the side where they "originated." If these different attended locations corresponded to stronger or weaker parts of the RF-for example, if the dot patch were not placed perfectly within the center of the RF_-such a scenario could mimic direction selectivity. In general, we could never be completely assured that the dot patch was perfectly centered in the RF, and in some cases, we found that the dot patch had been centered better than others (examples in Fig. 10A). Thus it is worth determining whether the direction selectivity of LIP neurons might have been related

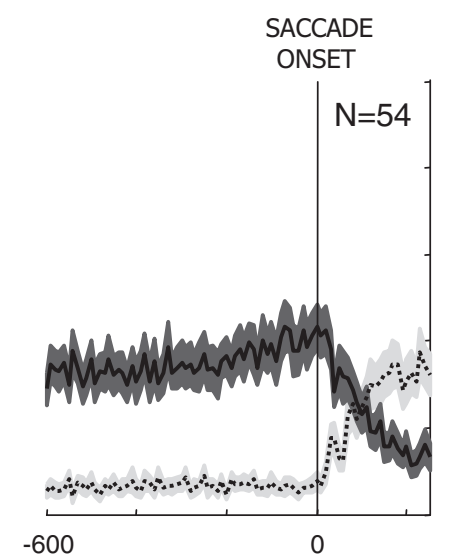

to the exact placement of the dot patch relative to the RF center.

To examine this issue, we analyzed the direction selectivity for each neuron in relation to the smoothed RF map generated using the RF-mapping technique (see METHODS). For each cell, we measured the distance between the center of the dot patch used for that cell and the center of the interpolated RF (if the center could be estimated; see METHODS). We found no systematic relationship between this offset and the direction selectivity: the median offset was $2.5^{\circ}$ for both direction-selective ( $n=$ 83 ), and nondirectional cells $(n=52$; Fig. 10B). However, 
A
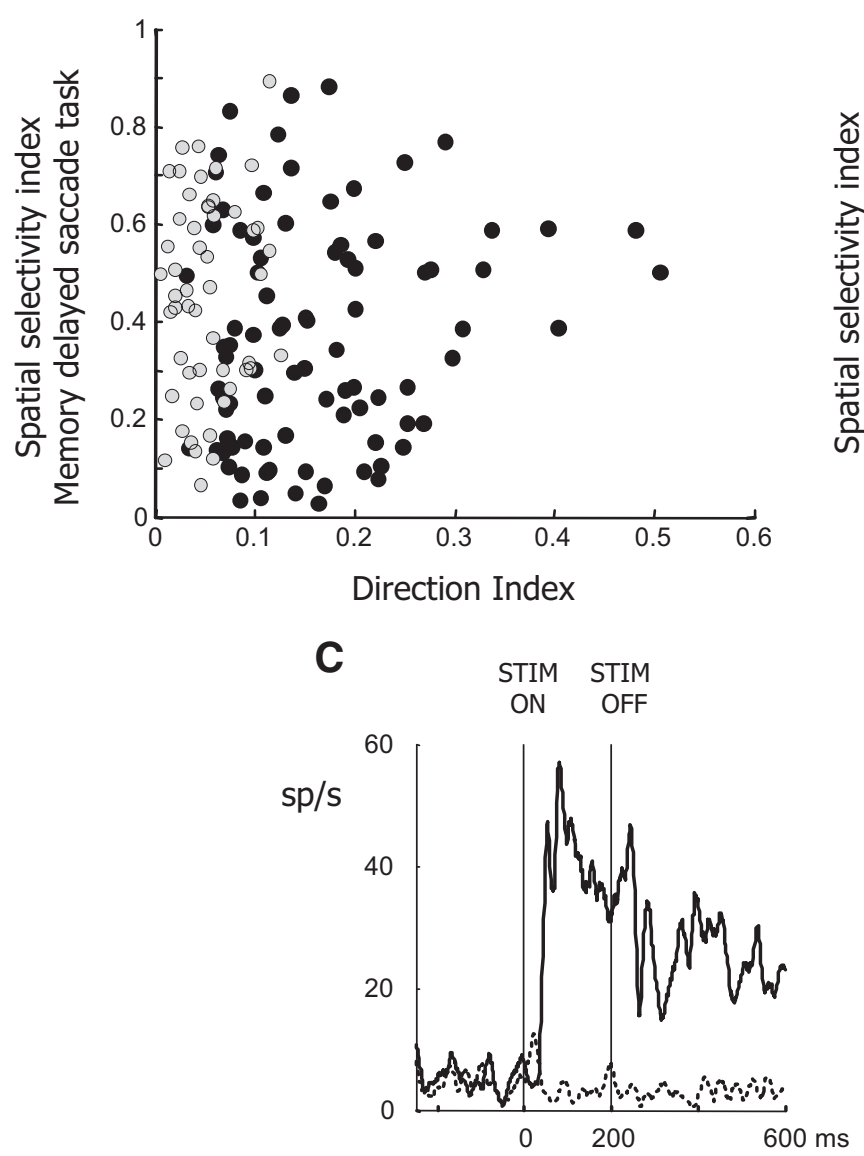

B
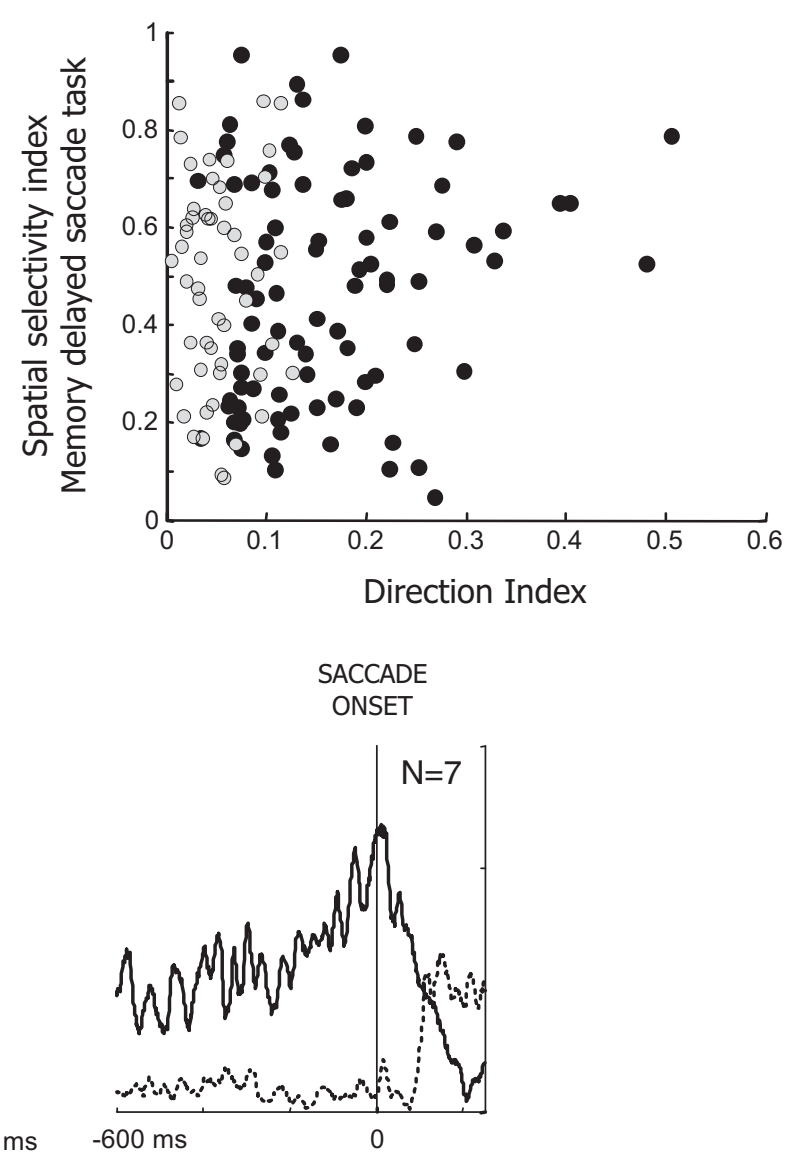

FIG. 9. Comparison between direction index in the main task and spatial selectivity indices for delay activity $(A)$ and presaccadic activity $(B)$ in the memory delayed saccade task, for all 143 LIP cells tested with both tasks. Delay activity was calculated over a 500-ms window starting 150 ms after the disappearance of the target, whereas the presaccadic activity was computed over the $200-\mathrm{ms}$ window before saccade onset. For each neuron, we calculated the spatial selectivity indices for the memory delayed saccade task using the same method that we used to calculate the DI (the normalized mean vector). Black and gray circles indicate direction-selective and non-direction-selective LIP cells, respectively. $C$ : population-average activity to the memory delayed saccade task for the 7 LIP cells with DI $>0.3$; same conventions as in Fig. 8. Responses were smoothed with a Gaussian filter with a SD of 25 ms.

even if the dot patch were centered perfectly within the RF center, the RFs of many LIP neurons are asymmetric. Although $\mathrm{RF}$ asymmetry per se should not affect visual responses to motion in different directions, it is hypothetically possible that the animals may attend to different sides of the dot patch depending on the direction of motion. If so, an RF asymmetry combined with spatial attention might mimic direction selectivity. To further examine any possible relationship between RF profile and direction selectivity, for each cell, we calculated a visual gradient index (VGI) from the smoothed RF map. The VGI was a normalized ratio of the strength of the RF at the two opposite sides of the dot patch corresponding to one axis of motion (see METHODS). We determined the maximum VGI ( $\max$ VGI) measured for each of the four axes of motion. If the animal hypothetically attended to different sides of the dot patch depending on the direction of motion, it would thus be expected that cells with larger max VGI would appear to be more "direction selective." However, for individual neurons, we found no relationship between the max VGI and direction selectivity. For example, the neuron on the left in Fig. 10A had a max VGI $=0.53$ and DI $=0.02$, whereas the neuron on the right had a max VGI $=0.27$ and $\mathrm{DI}=0.19$. Across all neurons, the distribution of max VGI values was not signifi- cantly different between direction-selective and nondirectional neurons (Fig. 10C; median VGI $=0.12$ and 0.15 , respectively; Wilcoxon rank sum test, $P>0.1$ ). Finally, across the 83 direction-selective cells, we found no systematic relationship between the preferred-null axis and the axis yielding the max VGI for each cell: the circular distribution of angular differences between the preferred-null axis and the axis yielding max VGI did not differ from a uniform distribution (Rayleigh test, $P>0.05$ ). Thus overall, the direction selectivity among our population of LIP neurons could not be explained by the spatial disposition of their static response fields.

\section{I S C U S S I ON}

We found that a majority $(\sim 60 \%)$ of LIP neurons are selective for the direction of motion within the RF. Many previous studies on LIP have shown that LIP neurons encode static locations; for example, LIP neurons may encode a plan to saccade to the RF location (Snyder et al. 2000) or indicate the general salience of the RF location (Goldberg et al. 2006). Before further considering the directional properties of LIP neurons, it is thus essential to examine whether the direction selectivity is genuine and not epiphenomenal to spatial encod- 
A

Max VGI $=0.53$

$\mathrm{DI}=0.02$

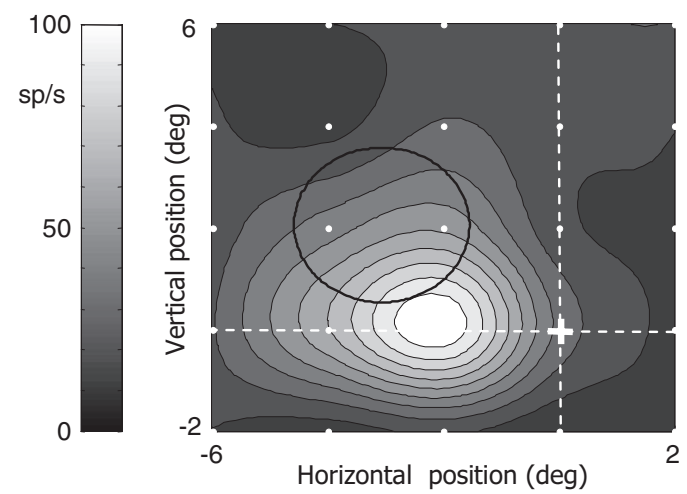

B

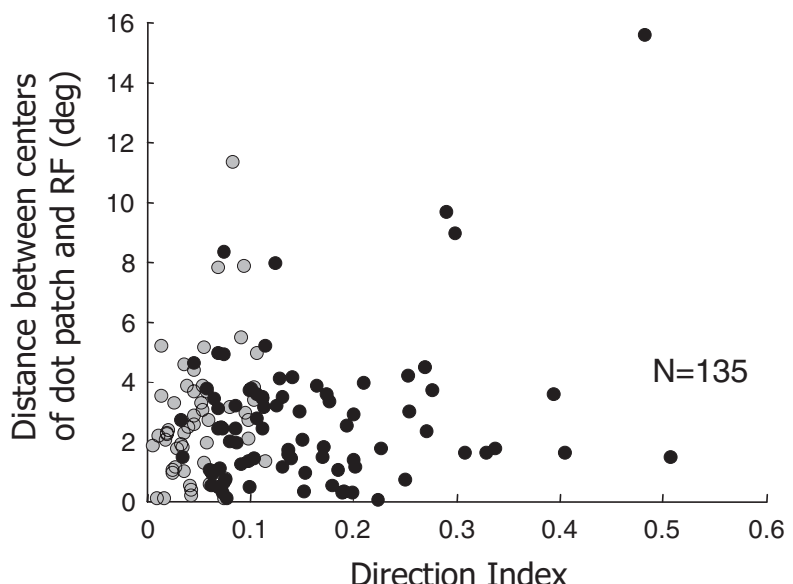

Max VGI $=0.27$

$\mathrm{DI}=0.19$

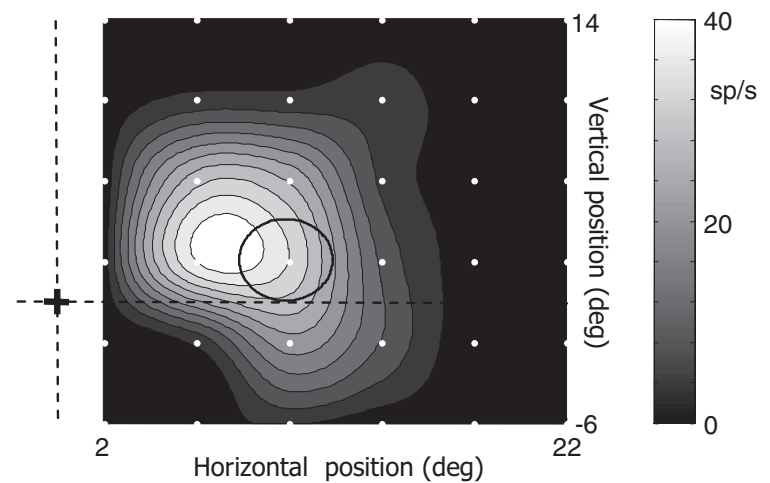

C

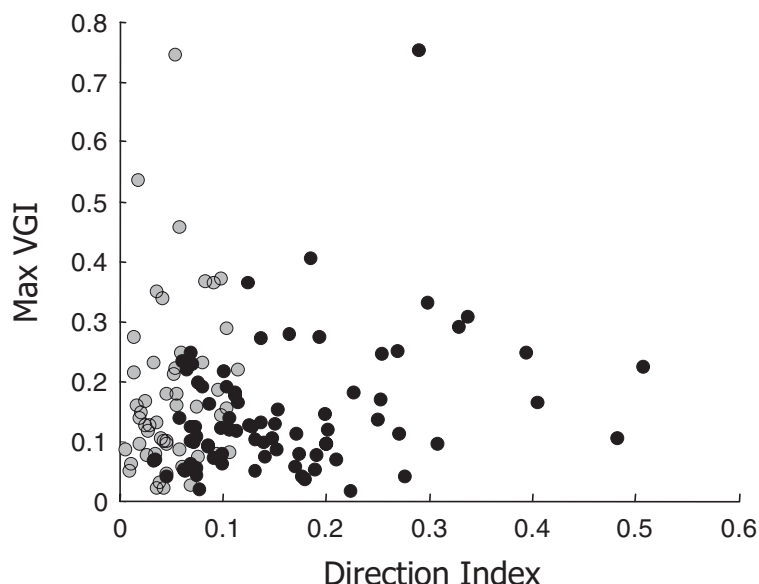

FIG. 10. A: RF map for a nondirectional LIP neuron (left) and a direction-selective LIP neuron (right). In both maps, the random dots patches (black circles)

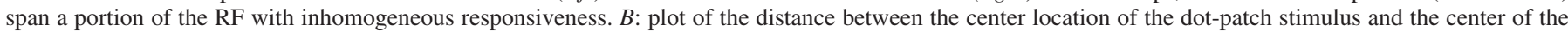

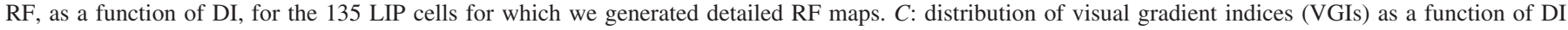

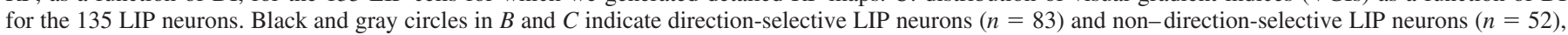
respectively.

ing. Under certain visual stimulus configurations, spatial encoding could masquerade as direction selectivity. For example, if a single spot of light is moved in opposite directions into or out of the RF of a neuron, the different responses of the neuron to the two directions could be misinterpreted as direction selectivity. To avoid confounds of this sort, we used patches of moving dots that always subtended the same part of visual space; this ensured that there was no physical spatial offset between different directions of motion. We also showed that asymmetries in the spatial RFs could not account for the directional properties of LIP neurons, in the hypothetical case that the animals reflexively attended to different parts of the motion patch depending on the direction of motion. Thus the direction selectivity that we found seems to be a bona fide property of LIP neurons and was not an artifact of spatial attention/planned eye movements or the way that the motion stimuli interacted with the spatial RF.

An important question is whether the direction selectivity we observed in LIP is a "normal" property of LIP neurons or rather arose because of the animals' task demands or training history. Several studies have shown that responses of LIP neurons can change depending on the requirements of the animals' task-athand (Stoet and Snyder 2004; Toth and Assad 2002). In our experiment, however, the animals had no task except to fixate during the presentation of the motion stimuli, and the direction of the stimuli was irrelevant to the animals' receiving reward. LIP responses can also be affected by the animals' long-term training (Freedman and Assad 2006; Grunewald et al. 1999; Law and Gold 2008). The two animals in this study had been exposed to moving visual stimuli in previous experiments (see METHODS), but those experiments did not require the discrimination of direction. Although we cannot rule out that the animals' previous exposure to moving stimuli per se may have strengthened direction selectivity in LIP, we believe that the direction selectivity was already present before the animals were trained in the laboratory. Our opinion is based partly on a previous study in which we showed that color selectivity was present in LIP in animals that had been trained to use color to select a saccade target but only when color was relevant to solving the task (Toth and Assad 2002). We stumbled onto that result because we originally did not find color selectivity in LIP with the animals passively fixating, even though color was 
relevant for performing the subsequent main task to receive reward. In contrast, in the case of direction selectivity in LIP, direction discrimination was not relevant for the animals' receiving reward, yet we still found direction selectivity during passive fixation. These findings favor the view that the direction selectivity in LIP was not a consequence of previous training or of exposure to moving stimuli in this study. Nonetheless, further experiments will be necessary to address this issue definitively.

The direction selectivity in LIP differs in some respects from that of other cortical areas in the dorsal visual pathway. For one, the width of direction-tuning curves was broader in LIP (median of $\left.136^{\circ}\right)$ than in MT $\left(\sim 90^{\circ}\right)$ (Maunsell and Van Essen 1983 ) but was comparable to that of VIP $\left(\sim 130^{\circ}\right)$ (Colby et al. 1993; Cook and Maunsell 2002). The average direction selectivity, as quantified by the DI, was also weaker in LIP than in MT or VIP (Colby et al. 1993; Maunsell and Van Essen 1983), although many individual LIP neurons were strongly directional.

The weaker average directionality in LIP may be caused in part by the interesting tonic offset in activity that was independent of direction. For example, in Fig. 5, the onset of the stationary dots caused a large transient response that decayed rapidly toward the baseline level of activity. However, the firing rate remained tonically elevated above the baseline level by a substantial amount ( $\sim 20$ spikes/s). When the dots began to move, there was a further modulation of firing rate about that tonic level: on average, motion in the preferred direction increased the firing rate above the tonic premovement firing rate, whereas motion in the null direction decreased the firing rate slightly below the tonic premovement rate (Fig. $5 B$ ). This suggests that the direction-selective response to motion is "superimposed" on the tonic response to the static dots in the RF. This pattern of activity is different from that found in other direction-selective areas, in which the response to static stimuli rapidly declines to near the baseline level (Movshon et al. 1990). Because we calculated the DI with respect to total firing rate during the motion period, a non-direction-selective tonic offset in activity would have reduced the measured DI. Indeed, when we subtracted the "baseline" activity before the start of motion, the mean normalized response ration was 0.73 , more comparable to that found for VIP neurons (0.86) (Colby et al. 1993) or MT neurons (0.93) (Maunsell and Van Essen 1983) (1.05) (Albright 1984). However, the median breadth of direction-tuning curves in LIP, which we measured independently of the offset, was still substantially broader than that found in areas such as MT.

The mechanism of the tonic offset of activity for LIP neurons is not known, but it is tempting to speculate that the source of the tonic activity may be distinct from the source of the direction-selective modulation. For example, LIP receives inputs from the ventral visual stream, including from area V4 and inferotemporal cortex (Andersen et al. 1990; Blatt et al. 1990; Lewis and Van Essen 2000a). It is possible that sloweradapting ventral pathway inputs directly or indirectly contribute to the tonic response of LIP neurons to the static dots, whereas dorsal pathway inputs, such as those from MT and MST, drive the direction-selective modulation that apparently superimposes on the tonic activity. The tonic offset could also reflect the animals' reflexive attention to the stimulus in the RF or a covert plan to saccade to the RF. Regardless, the pattern of activity that we observed suggests that our finding directionselective signals in LIP is perfectly compatible with previous results emphasizing that LIP neurons encode static locations. The tonic activity that we observed may signal the salience of (or intent to move the eyes to) the RF location when there is any visual stimulus in the RF, whereas the directionselective activity may be an orthogonal signal superimposed on the spatial signal.

In a previous study, Kusunoki et al. (2000) tested a small number (25) of LIP neurons with a single spot of light that moved through the RF in different directions. The LIP neurons responded to the motion, but the responses were not appreciably direction selective. The authors thus suggested that motion responses in LIP reflect the inherent salience of visual motion within the RF, regardless of direction. However, Kusunoki et al. only analyzed the first $200 \mathrm{~ms}$ of the responses to motion. In our experiment, we observed that the initial, transient response to the start of motion was less directional (Fig. 5B) and that, following motion onset, the direction selectivity developed over $\sim 200 \mathrm{~ms}$ (Fig. 5C). Thus presumably because we used a more sustained motion stimulus within the RF, we found a more prominent direction-selective response to motion. This sustained direction-selective response is not likely related to salience, because different neurons had different preferred directions and because motion in the RF should be salient regardless of direction. However, the less directionselective transient response at the onset of motion may be related to motion salience.

To our knowledge, there is little published information on the time course of direction selectivity in other cortical areas of the dorsal visual stream. Pack and Born (1999) reported that MT neurons also have a large nonspecific transient response at the start of motion and that direction selectivity in MT is likewise slow to develop following motion onset, on the order of $150 \mathrm{~ms}$. Although one must be cautious in comparing the two studies, it is interesting that the time course of direction selectivity that we found for LIP neurons is apparently not dramatically slower than that for MT neurons.

Although our study establishes that direction selectivity is common in LIP, many questions remain concerning the direction selectivity. For one, we only used translational motion in our study, but many neurons in MST, an area that projects to LIP, are selective for nontranslational patterns of optic flow, including rotation, expansion, and contraction (Duffy and Wurtz 1991; Graziano et al. 1994; Tanaka and Saito 1989). It is possible that some of the $\sim 40 \%$ of LIP neurons that were not selective for the direction of translational motion may be selective for other configurations of motion. The foveal or central bias that we found for preferred directions in LIP may also be indicative of a preference for more complex patterns of motion, even for neurons that were direction selective for translational motion. We also did not systematically test whether the direction selectivity is uniform throughout the RF. However, we found that most LIP neurons were similarly directional to patches of motion or to single spots of light traversing the RF, suggesting that the direction selectivity is likely to be uniform throughout the RF. These issues need to be addressed in more detail in future experiments.

Other outstanding issues are the origin and functional organization of direction selectivity in LIP. Lewis and Van Essen (2000a) reported that the heavily myelinated ventral subdivi- 
sion of LIP is preferentially innervated by MT and MST, areas that contain a preponderance of direction-selective neurons. Direction selectivity may be conferred to LIP by these inputs. Although we did not find any obvious asymmetries in the proportion of direction-selective neurons throughout the depth of LIP, this issue clearly needs to be examined in more detail. One pertinent observation is that we did not identify a subpopulation of neurons that was "specialized" for discriminating direction: the direction-selective neurons and non-directionselective neurons responded broadly similarly to the memory delayed saccade task (Figs. 8 and 9). In addition, we did not find any obvious clustering or columnar organization with respect to preferred direction in LIP, although our experiment was not optimized to show such clustering (e.g., we assessed each neuron with several tasks besides examining their direction tuning, which limited the number of neurons that could be recorded during a single daily electrode penetration through LIP). Nonetheless, it will be important to develop a coherent picture of the overall functional organization of LIP. As a start, it will be useful to examine more closely whether there are differences in the prevalence of direction-selective neurons between anatomically defined subdivisions within LIP (Lewis and Van Essen 2000a,b), as has been suggested for spatial selectivity (Ben Hamed et al. 2001; Blatt et al. 1990).

A concluding question might be the following: why have yet another direction-selective area within parietal cortex? Does LIP's role in motion processing differ from other nearby cortical areas, such as MT, MST, and VIP? Although we can only speculate at this point, several possibilities seem noteworthy. First, LIP has been shown to play an important role in spatial attention and/or eye movement planning, and LIP communicates directly with oculomotor structures, such as the frontal eye fields and the superior colliculus (Andersen et al. 1990). It thus seems inescapable that LIP is involved in assigning significance to locations in the visual world and in driving or biasing the eyes toward those locations. If so, information about direction could be essential for LIP to update or predict the location of moving visual targets or to trigger movements at appropriate times to acquire moving targets. Thus directional responses in LIP could be more related to how motion will be used by the animal than to how direction is computed per se.

A second (and related) possibility is that motion processing in LIP seems to be particularly subject to nonvisual influences. For example, in our laboratory, we showed that many LIP neurons, but not MST neurons, fire in a direction-selective manner during periods of occluded motion (while the animal is fixating) or make predictions about upcoming directions of motion (Eskandar and Assad 1999, 2002). We also showed that the firing of LIP neurons, but not MT or MST neurons, is closely aligned to the subjective perception of direction when direction is ambiguous (Williams et al. 2003). These findings suggest a general transformation within parietal cortex from a more veridical representation of retinal motion toward a representation more related to the way that the animal will interpret or make use of motion. In this view, the directional representations of motion in LIP might be expected to be more flexible, because the way that motion is used can vary with the demands of the task at hand. We recently tested this idea by training animals to group motion directions into two "artificial" categories. Following training, we found that LIP neurons showed a strong tendency to reflect the motion categories: many neurons responded similarly to directions within the categories but discriminated sharply between the categories. In contrast, whereas most MT neurons were direction selective in the categorization task, the distribution of their preferred directions showed no special relationship to the category boundary (Freedman and Assad 2006). The emerging picture is that areas such as MT may provide a fairly faithful representation of direction that is largely independent of task demands (although see Martinez-Trujillo and Treue 2004), whereas areas such as LIP encode direction in a way that varies with task demands. However, as we show here, direction-selective responses in LIP are present, independent of task demands. This direction selectivity may reflect unbiased direction-selective inputs to LIP from areas such as MT. Thus an intriguing hypothesis is that the plastic directional representation in areas such as LIP could be molded by dynamic or long-term modulation of inputs from areas such as MT (Ferrera and Grinband 2006).

\section{G R A N T S}

This work was supported by National Eye Institute Grant EY-12106.

\section{REFEREN C ES}

Albright TD. Direction and orientation selectivity of neurons in visual area MT of the macaque. J Neurophysiol 52: 1106-1130, 1984.

Andersen RA. The role of the inferior parietal lobule in spatial perception and visual-motor integration. In: The Handbook of Physiology. Section I: The Nervous System Volume V. Higher Functions of the Brain Part 2, edited by Plum F, Mountcastle VB, Geiger SR. Bethesda, MD: American Physiological Society, 1987, p. 483-518.

Andersen RA, Asanuma C, Essick G, Siegel RM. Corticocortical connections of anatomically and physiologically defined subdivisions within the inferior parietal lobule. J Comp Neurol 296: 65-113, 1990.

Andersen RA, Essick GK, Siegel RM. Encoding of spatial location by posterior parietal neurons. Science 230: 456-458, 1985.

Barash S, Bracewell RM, Fogassi L, Gnadt JW, Andersen RA. Saccaderelated activity in the lateral intraparietal area. I. Temporal properties; comparison with area 7a. J Neurophysiol 66: 1095-1108, 1991.

Ben Hamed S, Duhamel JR, Bremmer F, Graf W. Representation of the visual field in the lateral intraparietal area of macaque monkeys: a quantitative receptive field analysis. Exp Brain Res 140: 127-144, 2001.

Bisley JW, Goldberg ME. Neuronal activity in the lateral intraparietal area and spatial attention. Science 299: 81-86, 2003.

Blatt GJ, Andersen RA, Stoner GR. Visual receptive field organization and cortico-cortical connections of the lateral intraparietal area (area LIP) in the macaque. J Comp Neurol 299: 421-445, 1990.

Colby CL, Duhamel JR, Goldberg ME. Ventral intraparietal area of the macaque: anatomic location and visual response properties. J Neurophysiol 69: 902-914, 1993.

Colby CL, Duhamel JR, Goldberg ME. Visual, presaccadic, and cognitive activation of single neurons in monkey lateral intraparietal area. J Neurophysiol 76: 2841-2852, 1996.

Cook EP, Maunsell JH. Attentional modulation of behavioral performance and neuronal responses in middle temporal and ventral intraparietal areas of macaque monkey. J Neurosci 22: 1994-2004, 2002.

Crist CF, Yamasaki DS, Komatsu H, Wurtz RH. A grid system and a microsyringe for single cell recording. J Neurosci Methods 26: 117-122, 1988

Duffy CJ, Wurtz RH. Sensitivity of MST neurons to optic flow stimuli. I. A continuum of response selectivity to large-field stimuli. J Neurophysiol 65: 1329-1345, 1991.

Duhamel JR, Colby CL, Goldberg ME. The updating of the representation of visual space in parietal cortex by intended eye movements. Science 255 : 90-92, 1992.

Eskandar EN, Assad JA. Dissociation of visual, motor and predictive signals in parietal cortex during visual guidance. Nat Neurosci 2: 88-93, 1999.

Eskandar EN, Assad JA. Distinct nature of directional signals among parietal cortical areas during visual guidance. J Neurophysiol 88: 1777-1790, 2002. 
Ferrera VP, Grinband J. Walk the line: parietal neurons respect category boundaries. Nat Neurosci 9: 1207-1208, 2006.

Fisher NI. Statistical Analysis of Circular Data. New York: Cambridge University Press, 1993.

Freedman DJ, Assad JA. Experience-dependent representation of visual categories in parietal cortex. Nature 443: 85-88, 2006.

Goldberg ME, Bisley JW, Powell KD, Gottlieb J. Saccades, salience and attention: the role of the lateral intraparietal area in visual behavior. Prog Brain Res 155: 157-175, 2006.

Gottlieb JP, Kusunoki M, Goldberg ME. The representation of visual salience in monkey parietal cortex. Nature 391: 481-484, 1998.

Graziano MS, Andersen RA, Snowden RJ. Tuning of MST neurons to spiral motions. J Neurosci 14: 54-67, 1994.

Grunewald A, Linden JF, Andersen RA. Responses to auditory stimuli in macaque lateral intraparietal area. I. Effects of training. J Neurophysiol 82: 330-342, 1999.

Hikosaka O, Wurtz RH. Visual and oculomotor functions of monkey substantia nigra pars reticulata. III. Memory-contingent visual and saccade responses. J Neurophysiol 49: 1268-1284, 1983.

Janssen P, Shadlen MN. A representation of the hazard rate of elapsed time in macaque area LIP. Nat Neurosci 8: 234-241, 2005.

Judge SJ, Richmond BJ, Chu FC. Implantation of magnetic search coils for measurement of eye position: an improved method. Vision Res 20: 535-538, 1980.

Komatsu H, Wurtz RH. Relation of cortical areas MT and MST to pursuit eye movements. III. Interaction with full-field visual stimulation. J Neurophysiol 60: 621-644, 1988.

Kusunoki M, Gottlieb J, Goldberg ME. The lateral intraparietal area as a salience map: the representation of abrupt onset, stimulus motion, and task relevance. Vision Res 40: 1459-1468, 2000.

Law CT, Gold JI. Neural correlates of perceptual learning in a sensory-motor, but not a sensory, cortical area. Nat Neurosci 11: 505-513, 2008.

Lewis JW, Van Essen DC. Corticocortical connections of visual, sensorimotor, and multimodal processing areas in the parietal lobe of the macaque monkey. J Comp Neurol 428: 112-137, 2000a.

Lewis JW, Van Essen DC. Mapping of architectonic subdivisions in the macaque monkey, with emphasis on parieto-occipital cortex. J Comp Neurol 428: 79-111, 2000 b.

Maimon G, Assad JA. A cognitive signal for the proactive timing of action in macaque LIP. Nat Neurosci 9: 948-955, 2006.

Martinez-Conde S, Macknik SL, Hubel DH. Microsaccadic eye movements and firing of single cells in the striate cortex of macaque monkeys. Nat Neurosci 3: 251-258, 2000.

Martinez-Trujillo JC, Treue S. Feature-based attention increases the selectivity of population responses in primate visual cortex. Curr Biol 14: 744-751, 2004.

Maunsell JH, Van Essen DC. Functional properties of neurons in middle temporal visual area of the macaque monkey. I. Selectivity for stimulus direction, speed, and orientation. J Neurophysiol 49: 1127-1147, 1983.

McAdams CJ, Maunsell JH. Effects of attention on orientation-tuning functions of single neurons in macaque cortical area V4. J Neurosci 19: 431-441, 1999 .
Mesulam MM. Spatial attention and neglect: parietal, frontal and cingulate contributions to the mental representation and attentional targeting of salient extrapersonal events. Philos Trans R Soc Lond B Biol Sci 354: 1325-1346, 1999.

Movshon JA, Lisberger SG, Krauzlis RJ. Visual cortical signals supporting smooth pursuit eye movements. Cold Spring Harb Symp Quant Biol 55: 707-716, 1990.

Oristaglio J, Schneider DM, Balan PF, Gottlieb J. Integration of visuospatial and effector information during symbolically cued limb movements in monkey lateral intraparietal area. J Neurosci 26: 8310-8319, 2006.

Pack C, Born RT. Latency of direction tuning in area MT of awake behaving macaque. Soc Neurosci Abstr 25: 673, 1999.

Platt ML, Glimcher PW. Neural correlates of decision variables in parietal cortex. Nature 400: 233-238, 1999.

Robinson DA. A method of measuring eye movement using a scleral search coil in a magnetic field. IEEE Trans Biomed Eng 10: 137-145, 1963.

Sereno AB, Amador SC. Attention and memory-related responses of neurons in the lateral intraparietal area during spatial and shape-delayed match-tosample tasks. J Neurophysiol 95: 1078-1098, 2006.

Sereno AB, Maunsell JH. Shape selectivity in primate lateral intraparietal cortex. Nature 395: 500-503, 1998.

Snowden RJ, Treue S, Andersen RA. The response of neurons in areas V1 and MT of the alert rhesus monkey to moving random dot patterns. Exp Brain Res 88: 389-400, 1992.

Snyder LH, Batista AP, Andersen RA. Coding of intention in the posterior parietal cortex. Nature 386: 167-170, 1997.

Snyder LH, Batista AP, Andersen RA. Intention-related activity in the posterior parietal cortex: a review. Vision Res 40: 1433-1441, 2000.

Stoet G, Snyder LH. Single neurons in posterior parietal cortex of monkeys encode cognitive set. Neuron 42: 1003-1012, 2004.

Sugrue LP, Corrado GS, Newsome WT. Matching behavior and the representation of value in the parietal cortex. Science 304: 1782-1787, 2004.

Tanaka K, Saito H. Analysis of motion of the visual field by direction, expansion/contraction, and rotation cells clustered in the dorsal part of the medial superior temporal area of the macaque monkey. $J$ Neurophysiol 62 : 626-641, 1989.

Toth LJ, Assad JA. Dynamic coding of behaviourally relevant stimuli in parietal cortex. Nature 415: 165-168, 2002.

Treue S, Martinez Trujillo JC. Feature-based attention influences motion processing gain in macaque visual cortex. Nature 399: 575-579, 1999.

Ungerleider LG, Desimone R. Cortical connections of visual area MT in the macaque. J Comp Neurol 248: 190-222, 1986.

Williams ZM, Elfar JC, Eskandar EN, Toth LJ, Assad JA. Parietal activity and the perceived direction of ambiguous apparent motion. Nat Neurosci 6: 616-623, 2003.

Zaksas D, Pasternak T. Directional signals in the prefrontal cortex and in area MT during a working memory for visual motion task. J Neurosci 26: 11726-11742, 2006.

Zhang T, Heuer HW, Britten KH. Parietal area VIP neuronal responses to heading stimuli are encoded in head-centered coordinates. Neuron 42: 993-1001, 2004. 